Canadian University Music Review

Revue de musique des universités canadiennes

\title{
Vers une approche critique de la théorie schenkérienne
}

\section{Carmen Sabourin}

Numéro 15, 1995

URI : https://id.erudit.org/iderudit/1014391ar

DOI : https://doi.org/10.7202/1014391ar

Aller au sommaire du numéro

\section{Éditeur(s)}

Canadian University Music Society / Société de musique des universités canadiennes

\section{ISSN}

0710-0353 (imprimé)

2291-2436 (numérique)

Découvrir la revue

\section{Citer cet article}

Sabourin, C. (1995). Vers une approche critique de la théorie schenkérienne. Canadian University Music Review / Revue de musique des universités canadiennes, (15), 1-43. https://doi.org/10.7202/1014391ar

\section{Résumé de l'article}

Cette étude présente en premier lieu certains des éléments qui caractérisent les nouveaux espaces de réflexion en musicologie et en théorie de la musique. Puis, après avoir situé l'évolution récente de la théorie schenkérienne dans le panorama intellectuel, l'auteure expose une problématique qui vise à mettre en évidence la nécessité d’une interprétation éclairée de la réception et de l'appropriation des idées du théoricien autrichien Heinrich Schenker par les théoriciens-analystes anglo-américains. L'auteure suggère que la diversité et la complexité des paramètres en jeu dans la construction du discours schenkérien contemporain démontrent à l'évidence que seule une stratégie interprétative puissante pourra rendre compte de la richesse d'un phénomène aussi dense. Elle conclut en proposant que, face à la complexité de ce phénomène, il semble que seul le recours à des modèles de réflexion développés dans le domaine des sciences sociales et de l'anthropologie culturelle, et repris par les ethnomusicologues, permettra de formuler une révision critique du discours schenkérien.
All Rights Reserved @ Canadian University Music Society / Société de musique des universités canadiennes, 1995
Ce document est protégé par la loi sur le droit d'auteur. L’utilisation des services d'Érudit (y compris la reproduction) est assujettie à sa politique d'utilisation que vous pouvez consulter en ligne.

https://apropos.erudit.org/fr/usagers/politique-dutilisation/ 


\section{VERS UNE APPROCHE CRITIQUE DE LA THÉORIE SCHENKÉRIENNE}

\section{Carmen Sabourin}

Lors d'un échange récent avec un théoricien d'obédience formaliste ${ }^{1}$ au sujet de la pertinence de la théorie schenkérienne en ce qui concerne l'analyse de la musique tonale française, j'ai eu droit à l'ultime remontrance. Comment osaisje douter de l'applicabilité de la théorie de Schenker à quelque musique tonale que ce soit? Selon mon interlocuteur, « l'approche de Schenker est internationale et universelle; elle s'applique non seulement à la musique allemande, mais à la musique italienne, russe, polonaise, tchèque, etc. » La simple suggestion que des modifications des paradigmes schenkériens pourraient être nécessaires pour rendre compte de la spécificité de la grammaire tonale française révèlait à ses yeux une " profonde incompréhension des idées de Schenker ». En effet, comment osais-je prétendre que la musique française puisse être porteuse d'une quelconque différence, d'une spécificité bien à elle du point de vue du traitement de la tonalité ? Toujours selon mon interlocuteur, «en dépit de l'influence des théories de Rameau, la musique française, de Rameau lui-même à Fauré et à Debussy, peut être comprise en termes schenkériens sans modifier l'approche de Schenker. »

Qu'est-ce qui indisposait tant mon interlocuteur dans l'hypothèse de l'inadéquation de la théorie schenkérienne pour la musique française ? Deux énoncés se dégagent de son propos. Le premier : l'approche de Schenker est universelle parce qu'elle est à même d'expliquer toutes les musiques tonales ; le deuxième : l'approche de Schenker ne doit pas être modifiée parce que la musique tonale est universelle, quelles que soient la nationalité du compositeur et l'époque à laquelle l'œuvre a été créée. À cet égard, le lecteur conviendra que mon interlocuteur est prodigue en délimitant le champ schenkérien en plaçant Rameau à une extrémité et Fauré et Debussy à l'autre. Les énoncés ci-dessus établissent en quelque sorte une relation d'identité entre la théorie schenkérienne et le système tonal. Comme le confirme la définition de la tautologie, le prédicat ici ne nous apprend rien de plus que le sujet. La tautologie étant un truisme, une vérité d'évidence, que peut-on ajouter à l'argument d'universalité invoqué ici ?

1 Au sens précis de celui qui se concentre spécifiquement sur les structures internes des œuvres conçues en tant que phénomènes autonomes, soit sans relation avec le contexte social, politique et culturel dans lequel elles ont été composées. 
Ce qui d'emblée étonne chez mon interlocuteur (qui n'est pas lui-même un praticien de l'analyse schenkérienne), c'est cet empressement à affirmer l'infaillibilité des idées de Schenker, de même qu'à niveler, à uniformiser toute spécificité, toute différence potentielle dans le traitement de la tonalité en tant que système de pensée, que ce soit d'un compositeur à l'autre, d'une nationalité à l'autre ou d'une époque à une autre. Que révèle donc cet empressement, qu'y a-t-il donc à gagner dans la perpétuation de la notion d'universalité d'une théorie, surtout en cette fin de siècle postmoderne où l'on assiste à une remise en question des valeurs au cœur du mouvement philosophique des Lumières : humanisme, histoire, progrès, liberté, raison, science ? Pourquoi un théoricien de la musique devrait-il rester au-dessus de la mêlée ? Qu'est-ce donc qui le motive à ignorer que cette remise en question a provoqué une série de crises ressenties par les intellectuels comme la fin des grands récits qui légitimaient les discours historiques, culturels, sociaux et scientifiques du projet moderniste ? La question de la perpétuation des notions d'universalité et d'infaillibilité d'une théorie est riche de nombreuses ramifications ; elle nous projette en plein cœur des débats qui animent depuis quelques années la scène musicologique anglo-américaine. L'art d'argumenter de mon interlocuteur n'est certes pas représentatif de la manière dont les débats y sont menés, mais son propos révèle un aspect des tensions qui bouleversent et concourent à transformer la discipline de la musicologie depuis les 10 dernières années et, plus récemment, celle de la théorie.

Je propose ici une réflexion dont la valeur heuristique contribuera, je l'espère, à alimenter le débat sur l'avenir de la théorie schenkérienne. Dans un premier temps, je présenterai certains des éléments qui caractérisent les nouveaux espaces de réflexion en musicologie et en théorie de la musique. En second lieu, je porterai mon attention sur la situation qui prévaut au sein de la discipline de la théorie en ce qui concerne l'analyse schenkérienne. Puis, j'exposerai une problématique visant à mettre en évidence la nécessité d'une interprétation éclairée de la réception et de l'appropriation des idées du théoricien autrichien Heinrich Schenker par les théoriciens-analystes angloaméricains.

\section{Musicologie}

La musicologie anglo-américaine traditionnelle, définie en 1985 par Joseph Kerman en tant que discipline «traitant essentiellement du factuel, du documentaire, du vérifiable, de l'analysable, du positiviste ${ }^{2}$ », a été secouée au

2 «dealing essentially with the factual, the documentary, the verifiable, the analysable, the positivistic. " Musicology (Londres: Fontana, 1985), édition américaine sous le titre de 
cours de la dernière décennie par des chercheurs audacieux qui travaillent à l'élaboration d'un discours nouveau critique. Cette orientation critique est caractérisée par l'appropriation de modèles théoriques provenant de disciplines et de champs d'études aussi divers que la critique littéraire, le poststructuralisme, la déconstruction, la sémiologie, la critique féministe, l'ethnomusicologie, la sociologie, l'anthropologie culturelle, la psychanalyse et la philosophie.

Dès 1985, Kerman observait : «Ce que j'appellerais la critique musicale sérieuse - la critique musicale de type universitaire si vous préférez n'existe pas en tant que discipline au même titre que la musicologie et la théorie musicale d'une part, ou la critique littéraire et la critique d'art d'autre part ${ }^{3}$. » La situation a évidemment littéralement explosé depuis lors. Les nouvelles tendances, encore récemment associées à la musicologie dite nouvelle, alternative ou marginale, sont en train de transformer radicalement la discipline. Dans le cadre d'une évaluation prospective de la situation en musicologie historique pour les années 1990, Kerman reconnaissait, statistiques à l'appui, que l'intégration des nouveaux paramètres constitue « une tendance authentique, et non pas un ensemble d'écrits isolés qui d'aventure n'intéressent qu'une personne en particulier ${ }^{4}$. »

Cette orientation critique s'inscrit dans le sillage des courants de réflexion qui ont émergé progressivement à la faveur du développement, fort complexe,

Contemplating Music: Challenges to Musicology (Cambridge, Mass. : Harvard University Press, 1985), 12.

3 « What I would call serious music criticism - academic criticism, if you prefer - does not exist as a discipline on a par with musicology and music theory on the one hand, or literary and art criticism on the other." Kerman, Musicology, 17. Toutes les traductions sont de l'auteur. Musicology de Kerman constitue un véritable plaidoyer invitant la communauté des chercheurs à intégrer le champ de la critique musicale à l'entreprise musicologique. Tout en rappelant que la critique musicale a toujours été pratiquée par un petit groupe de musicologues (notamment Charles Rosen, Leonard B. Meyer, Edward T. Cone, Leo Treitler et Maynard Solomon), Kerman expose les conditions historiques qui ont contribué à maintenir les disciplines de la musicologie et de la théorie hors du champ de la critique musicale. Cet ouvrage aura été un catalyseur et ainsi le point de départ de nombreuses contributions dans le domaine. Voir le compte rendu de Leo Treitler, « The Power of Positivist Thinking ", Journal of the American Musicological Society 42, $\mathrm{n}^{\circ} 2$ (1989): 375-402. Pour une approche critique du projet de Kerman, voir John Shepherd, "Vers une critique sociologiquement fondée de la musique », Alternative Musicologies/Les musicologies alternatives, numéro spécial de la Revue de musique des universités canadiennes, $\mathrm{n}^{\circ}$ 10/2 (1990): 48-67.

4 « a genuine trend, not just a collection of isolated writings that happen to interest one particular person. » Kerman, « American Musicology in the 1990s », The Journal of Musicology 9, $\mathrm{n}^{\circ} 2$ (1991): 131-43 ; 136. Pour un compte rendu des nouvelles tendances en musicologie et en théorie musicale, voir aussi Renée Lorraine, « Musicology and Theory : Where It's Been, Where It's Going », The Journal of Aesthetics and Art Criticism 51, nº 2 (1993): 235-44. 
du projet intellectuel des « cultural studies $»^{5}$. Ce projet ne constitue pas une discipline académique mais bien plutôt une attitude ou, mieux encore, une pratique au sein de laquelle l'étude des relations entre culture et société, et, plus spécifiquement, l'examen des forces historiques, politiques, sociales, économiques et culturelles qui façonnent l'expérience vécue aussi bien que les incidences de la réalité vécue sur ces mêmes forces sont, pour bon nombre des praticiens, inséparables d'un engagement politique dont le but avoué est « de comprendre et de changer les structures de domination dans les sociétés industrielles capitalistes ${ }^{6}$. » Les contributions au projet des « cultural studies » sont toutes caractérisées par une constante remise en question, révision, redéfinition des moyens et des fins du projet, et ce, qu'elles proviennent des tenants d'un discours politisé ou des tenants d'une approche consacrée davantage à la théorie. Enfin, ce projet propose des débats dont l'actualité et le dynamisme sont extrêmement stimulants et réjouissants, particulièrement pour le musicien qui se sera occasionnellement senti prisonnier de l'étroitesse et de l'artificialité inhérentes à certaines formes d'académisme.

Initialement, les modèles de pensée en vigueur dans le champ des « cultural studies » provenaient des domaines de la théorie littéraire et de l'anthropologie. Les transformations rapides et constantes des espaces politique et culturel au cours des dernières décennies ont évidemment engendré de nouveaux questionnements et, conséquemment, de nouvelles problématiques avec les développements théoriques afférents. On pense ici à la place relativement nouvelle qu'occupe l'étude de la musique populaire dans les disciplines de la musicologie, de l'ethnomusicologie et de la sociologie de la musique ${ }^{7}$. Comme le fait remarquer John Shepherd, « c'est dans le cadre des études sur la musique populaire qu'ont été traités avec le maximum d'acuité les problèmes liés au développement d'une critique musicale socialement fondée ${ }^{8}$. »

5 Pour un compte rendu du développement des "cultural studies », des origines en GrandeBretagne à leur évolution récente dans les communautés intellectuelles américaines et canadiennes-anglaises, voir l'essai de Lawrence Grossberg, «The Formations of Cultural Studies : An American in Birmingham ", dans Relocating Cultural Studies, éd. par Valda Blundell, John Shepherd et Ian Taylor (New York : Routledge, 1993), 21-66.

6 « to understand and change structures of dominance in industrial capitalist societies. » « Editors' Introduction ", dans Relocating Cultural Studies, 3.

7 Le lecteur pourra consulter Will Straw, « Review Essay », Alternative Musicologies/Les musicologies alternatives, numéro spécial de laRevue de musique des universités canadiennes, $\mathrm{n}^{\circ} 10 /$ 2 (1990) : 157-61; 157, où l'auteur commente quatre textes qui « représentent une entreprise de consolidation et un résumé de la recherche en musique populaire » (« represent an enterprise of consolidation and summary in popular music research $»)$.

8 Shepherd, «Vers une critique sociologiquement fondée de la musique », 53. 
On ne saurait s'étonner que certaines discipline se soient inscrites plus rapidement ou plus spontanément que d'autres dans le projet intellectuel des « cultural studies ». L'appropriation par les musicologues des objets d'étude, tels société, culture, idéologie, politique, canon, classe sociale, ethnicité, multiculturalisme, homosexualité, femmes, différence, sexes et sexualité est relativement récente par rapport au chemin parcouru dans d'autres domaines'. La complexité inhérente à l'élaboration d'une théorie des relations entre d'une part un ou plusieurs des objets d'étude qui sont au cœur des problématiques propres aux «cultural studies » et d'autre part la musique ne peut à elle seule expliquer le retard accusé par la musicologie. En effet, on ne saurait comprendre ce phénomène sans un examen attentif des enjeux sous-jacents à la perpétuation du statu quo pour la communauté des musicologues et sans la prise en considération des conditions historiques et culturelles propres au contexte universitaire anglo-américain. Il est à la fois inquiétant de constater que la musicologie ait mis tant de temps à faire siennes les préoccupations politiques et sociales de ce projet et réjouissant d'observer que cette même orientation critique est en plein épanouissement au sein de la discipline.

\section{Qu'en est-il de la théorie?}

Les nouveaux courants de réflexion qui animent la musicologie ne sont pas demeurés sans écho dans le domaine de la théorie. Au début des années 80, Joseph Kerman reprochait aux analystes de la génération post-Schenker et Tovey de n'avoir pas su, comme ces maîtres, concevoir l'analyse comme un « ajout essentiel à un système de valeur esthétique pleinement structuré » et, en fait, « d'avoir évité les jugements de valeur et adapté leur recherche au cadre des propositions strictement amendables, équations mathématiques, formulations du type théorie des ensembles, et leurs équivalents - tout ceci, apparemment, dans un effort pour acquérir le statut d'objectivité et dès lors

9 Voir, entre autres, Susan McClary, Feminine Endings : Music, Gender, and Sexuality (Minnesota : University of Minnesota Press, 1991), 5, sur le retard accusé par la discipline de la musicologie par rapport à la critique féministe : «I am painfully aware that this volume - one of the first books of feminist criticism in the discipline of musicology - is being assembled at a time when cynical voices in many other fields are beginning to declare feminism to be passé. It almost seems that musicology managed miraculously to pass directly from pre- to postfeminism without ever having to change - or even examine - its ways. " Voir aussi Philip V. Bohlman, «Musicology as a Political Act », The Journal of Musicology 11, n 4 (1993) : 411-36 ; 42930. L'auteur observe qu'en dépit du fait que le « rap » soit rapidement devenu un objet d'étude dans des disciplines telles la sociologie, l'anthropologie, la linguistique et les sciences politiques, sans parler des «African-American Studies » et de la théorie littéraire, il n'occupe toujours qu'une place marginale en musicologie. 


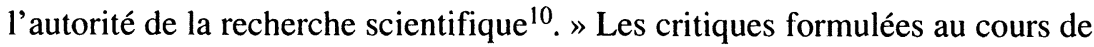
la dernière décennie par les musicologues ont engendré nombre de remises en question, dont principalement : (1) l'a priori selon lequel la musique est une structure autonome, en ce sens qu'elle peut être analysée sans égard au contexte social, politique, économique et culturel dans lequel elle a été composée et dans lequel elle est reçue ${ }^{11} ;(2)$ l'hypothèse voulant que la musicologie positiviste et la discipline de la théorie soient exemptes de tout parti pris idéologique ${ }^{12}$; (3) l'existence du canon compris ici dans le sens d'une institution qui pratique l'exclusion en ceci que le canon établit quelles œuvres sont dignes de l'intérêt des chercheurs en musicologie et en théorie ${ }^{13}$.

Ces remises en question, qui mettent toutes en évidence le développement d'une conscience critique au sein de la discipline de la musicologie, ont très souvent écorché au passage la systématisation excessive propre à la théorie de

10 « an essential adjunct to a fully articulated aesthetic value system »; « analysts have avoided value judgements and adapted their work to a format of strictly corrigible propositions, mathematical equations, set theory formulations, and the like - all this, apparently, in an effort to achieve the objective status and hence the authority of scientific inquiry. » Kerman, « How We Got Into Analysis, and How to Get Out », Critical Inquiry 7 (1980) : 311-31; 313.

11 Voir, entre autres, Janet Wolff, «The Ideology of Autonomous Art » dans Music and Society : The Politics of Composition, Performance and Reception, éd. par Richard Leppert et Susan McClary (Cambridge : Cambridge University Press, 1987), 1-12, qui examine l'origine et le développement au $\mathrm{XIX}^{\mathrm{c}}$ siècle de l'idéologie de la transcendance de toute activité artistique sous-jacente à la notion de musique en tant que structure autonome. Voir aussi Christine Battersby, Gender and Genius : Towards a Feminist Aesthetics (Londres : Women's Press, 1989), qui retrace l'évolution du concept de génie depuis l'antiquité jusqu'à nos jours et examine les conséquences de l'appropriation de la notion de génie par les artistes du XIX ${ }^{\mathrm{e}}$ siècle ; et Lydia Goehr, « Political Music and the Politics of Music », The Journal of Aesthetics and Art Criticism 52, n० 1 (1994) : 99-112.

12 Voir Rose Rosengard Subotnik, «The Role of Ideology in the Study of Western Music », paru initialement dans The Journal of Musicology 2, $\mathrm{n}^{\circ} 1$ (1983) : 1-12, et repris dans Developing Variations : Style and Ideology in Western Music (Minneapolis : University of Minnesota Press, 1991), 3-14, où elle démontre que prétendre échapper à toute idéologie est en soi une idéologie. Voir aussi Janet M. Levy, «Covert and Casual Values in Recent Writings About Music », The Journal of Musicology 5, $\mathrm{n}^{\circ} 1$ (1987) : 3-27.

13 Kerman, dans «A Few Canonic Variations », Critical Inquiry 10 (1983) : 107-25 ; 114, donne la définition suivante du canon telle qu' utilisée dans les arts en général : « an enduring exemplary collection of books, buildings, and paintings authorized in some way for contemplation, admiration, interpretation, and the determination of value. » Dans cet article, Kerman analyse les conditions idéologiques et historiques sous-jacentes à la formation du canon en musique et avance que "les répertoires sont déterminés par les interprètes, les canons, les critiques " (« repertories are determined by performers, canons, by critics »). Pour une contribution plus récente à ce sujet, voir Disciplining Music : Musicology and lts Canons, éd. par Katherine Bergeron et Philip V. Bohlman (Chicago : The University of Chicago Press, 1992). 
la musique ${ }^{14}$. Les théoriciens ont été accusés de fonctionner par raisonnement circulaire et de limiter leur entreprise à démontrer l'unité, la cohérence des œuvres analysées ${ }^{15}$ : les théories universalistes, les procédures analytiques utilisées et le choix des œuvres ou des extraits étudiés ne contribuant ultimement qu'à légitimer la théorie elle-même et à en assurer la viabilité, de même qu'à valider et à perpétuer le canon dans son principe d'exclusion. Dans un article sur la critique musicale où il examine l'origine de l'idéologie de l'organicisme et l'influence de cette idéologie sur les critiques et analystes du XIX ${ }^{e}$ siècle de même que sur les théoriciens-analystes anglo-américains contemporains, Kerman observe :

La vision de ces analystes-critiques était et demeure une vision impliquant une relation parfaite, organique entre toutes les parties analysable d'un chef-d'œuvre musical. Des techniques d'analyse de plus en plus sophistiquées tentent de montrer comment tous les aspects ou "paramètres » ou «domaines» du chef-d'œuvre remplissent leur fonction au profit de la structure totale. Des critiques qui differrent grandement les uns des autres par leurs méthodes, leurs styles et leurs forces voient toujours l'œuvre d'art en dernière instance comme un organisme dans ce sens. Selon le point de vue de l'idéologie dominante, l'analyse existe dans le but de démontrer l'organicisme, et l'organicisme existe dans le but de valider un certain corpus d'œuvres d'art ${ }^{16}$.

Les publications récentes dans le domaine de la théorie témoignent d'un certain besoin de redéfinir (sinon de définir) la discipline, comme en témoignent

14 Pour une prise de position radicale contre l'excès de systématisation en composition et en théorie, voir Susan McClary, «Terminal Prestige : The Case of Avant-Garde Music Composition », Cultural Critique 12 (1989) : 57-81.

15 Voir entre autres Richard Taruskin, recension de The Harmonic Structure of "The Rite of Spring " de Allen Forte, Current Musicology 28 (1979) : 114-29; et Alan Street, "Superior Myths, Dogmatic Allegories: The Resistance to Musical Unity », Music Analysis 8, $\mathrm{n}^{\text {os }} 1-2$ (1989) : 77-123.

16 " The vision of these analyst-critics was and is of a perfect, organic relation among all the analyzable parts of a musical masterpiece. Increasingly sophisticated techniques of analysis attempt to show how all aspects or "parameters" or "domains" of the masterpiece perform their function for the total structure. Critics who differ vastly from one another in their methods, styles, and emphases still view the work of art ultimately as an organism in this sense. From the standpoint of the ruling ideology, analysis exists for the purpose of demonstrating organicism, and organicism exists for the purpose of validating a certain body of works of art. " « How We Got into Analysis ", 315. Pour un examen de l'idéologie de l'organicisme, voir Ruth Solie, « The Living Work : Organicism and Musical Analysis », 19th-Century Music 4, $\mathrm{n}^{\circ} 2$ (1980) : 147-56. 
les entreprises suivantes: (1) le débat portant sur les relations entre les procédures explicatives utilisées en analyse musicale et les modèles scientifiques de recherche, paru dans le Journal of Music Theory (un des bastions de la théorie dite scientifique) et auquel six théoriciens ont participé17 ; (2) le collectif réunissant 19 théoriciens qui, à la demande des éditeurs de l'Indiana Theory Review, ont offert leur opinion sur le devenir de la théorie ${ }^{18}$; ou encore (3) le symposium intitulé Music Theory and Other Disciplines : Three Views ${ }^{19}$, qui associait trois théoriciens et trois chercheurs provenant de disciplines diverses (musicologie, philosophie et littérature) afin d'examiner et de contrer la tendance à marginaliser la théorie, tendance émanant de la musicologie interdisciplinaire qui suggère, « de manière implicite ou explicite, que l'on pourrait éluder le développement professionnel récent de la théorie de la musique et de l'analyse $\mathrm{e}^{20}$. » Ces entreprises expriment, chacune à sa manière, un certain sentiment qu'il y a péril en la demeure.

Une évaluation approfondie des différentes avenues de recherche en théorie de même que l'examen critique des nouveaux paradigmes et des entreprises interdisciplinaires qui fleurissent au sein de la discipline débordent évidemment

17 Matthew Brown et Douglas J. Dempster, «The Scientific Image of Music Theory », Journal of Music Theory 33, $\mathrm{n}^{\circ} 1$ (1989) : 65-106. Les auteurs amorcent leur réflexion en admettant candidement qu'il n'existe pas de consensus en ce qui concerne les fins et les méthodologies au sein de la discipline. Ils proposent un discours scientifique plus rigoureux que celui pratiqué jusqu'à ce jour par les théoriciens, qu'ils fondent sur un modèle scientifique d'explication auquel certaines modifications sont apportées, ( le modèle déductif-nomologique ou D-N mis au point dans les années 1950 et 1960 par Carl Hempel », p. 68). L'utilisation de ce modèle modifié de même que leur tentative de départager « particularistes » et « universalistes » ont été fortement critiquées par les quatre répondants: Benjamin Boretz, "The Logic of What ?", 107-16; Nicholas Cook, "Music Theory and "Good Comparison" : A Viennese Perspective ", 117 41 ; John Rahn, «Notes on Methodology in Music Theory», 143-54; Richard Taruskin, "Reply to Brown and Dempster », 155-64. Voir aussi la réponse de Dempster et de Brown aux répondants : "Evaluating Musical Analyses and Theories: Five Perspectives", Journal of Music Theory 34, $\mathrm{n}^{\circ} 2$ (1990) : 247-79.

18 Collection de 19 courts essais intitulée "The Future of Theory ", Indiana Theory Review 10 (1989) : 65-108.

19 Paru dans The Journal of Musicology $11, \mathrm{n}^{\circ} 1$ (1983). Ce symposium inclut les contributions suivantes : Fred Everett Maus, «Introduction », 6-10; J. Peter Burkholder, « Music Theory and Musicology », 11-23 ; Robert Hatten, « Response to Burkholder », 24-33 ; Kendall L. Walton, «Understanding Humor and Understanding Music », 32-44; Marion A. Guck, "Taking Notice: A Response to Kendall Walton », 45-51 ; Lawrence Rosenwald, "Theory, TextSetting, and Performance », 52-65 ; Fred Everett Maus, « Response to Lawrence Rosenwald », 66-72.

20 "with the suggestion, implicit or explicit, that one might sidestep the recent professional development of music theory and analysis. » Maus, «Introduction », 7. 
notre propos ${ }^{21}$. Toutefois, à la lecture des diverses opinions émises sur le devenir de la théorie, on peut constater que la majorité des théoriciens ne songent nullement à inscrire leur démarche dans un cadre socio-politique. La plupart résistent à l'idée de renoncer aux prémisses constitutives de la discipline, plus précisément, l'analyse des seules structures internes des œuvres prises hors de tout contexte, qu'il soit historique, économique, social ou autre, et l'élaboration de procédures explicatives qui se doivent sinon de constituer une véritable méthodologie scientifique, du moins de s'approcher, je dirais, plus ironiquement, de ressembler autant que faire se peut à une science. Cependant, la résistance au changement signifie bel et bien la présence d'une opposition à une force et, dès lors, la reconnaissance de cette force.

Sans vouloir sous-estimer les résultats, souvent extrêmement stimulants, du travail effectué par les théoriciens, ou déprécier les contributions tributaires d'outils d'analyse complexes, et surtout sans vouloir passer sous silence l'immense plaisir que procure l'analyse musicale, on peut se demander quelles sont les forces qui empêchent d'amorcer le décloisonnement, souhaité par plusieurs, d'une discipline qui a longtemps évolué dans le plus flagrant des isolements, à la fois par rapport à de nombreuses disciplines (littérature, sciences sociales, anthropologie, etc.) et, quoique depuis peu, par rapport à la musicologie. Il existe des amorces de réponse à cette question, généralement sous forme de critiques, et de critiques formulées principalement par les musicologues. Les Kerman, Treitler, Taruskin, et $\mathrm{McClar}^{22}$, pour ne nommer

21 Pour un compte rendu des nouvelles avenues de recherche en théorie de la musique, voir entre autres : Mary Louise Serafine et Wayne Slawson, «Interdisciplinary Directions in Music Theory »,Music Theory Spectrum 11 (1989) : 74-83 ; John Rahn, « New Research Paradigms », Music Theory Spectrum 11 (1989) : 84-94; Renée Lorraine, « Musicology and Theory : Where It's Been, Where It's Going ", The Journal of Aesthetics and Art Criticism 51, $\mathrm{n}^{\circ} 2$ (1993) : 23544. Un sujet de plus en plus discuté par les musicologues et les théoriciens qui privilégient une approche interdisciplinaire est celui de la narrativité en musique ; voir entre autres Carolyn Abbate, Unsung Voices : Studies of Opera and Musical Narratives in the Nineteenth Century (Princeton : Princeton University Press, 1991) ; Lawrence Kramer, Music as Cultural Practice, 1800-1900 (Berkeley : University of California Press, 1990) ; Anthony Newcomb, « Schumann and Late Eighteenth-Century Narrative Strategies ", 19th-Century Music 11, n⿳2 2 (1987) : 16474 ; et les contributions au débat de Fred Everett Mauss et Patrick McCreless. Les nouveaux développements en sémiologie incluent deux contributions importantes : V. Kofi Agawu,Playing with Signs : A Semiotic Interpretation of Classic Music (Princeton : Princeton University Press, 1991), et Jean-Jacques Nattiez, Musicologie générale et sémiologie (Paris : Christian Bourgois, 1987), traduit par Carolyn Abbate sous le titre Music and Discourse : Toward a Semiology of Music (Princeton : Princeton University Press, 1990).

22 Pour un aperçu du type de réaction qu'engendrent à l'occasion ces critiques chez les théoriciens, voir Pieter C. van den Toorn, "Politics, Feminism, and Contemporary Music Theory », The Journal of Musicology 11, n 3 (1991) : 275-99. 
que ceux-là, ont tous pris position face aux excès de systématisation propres à la théorie et face à son isolement par rapport à la vie intellectuelle en général.

\section{Qu'en est-il de la théorie schenkérienne ?}

Si les tentatives d'expliquer les motifs sous-jacents à la construction d'une « image scientifique de la théorie de la musique » provenant de l'intérieur de la discipline sont pratiquement inexistantes ${ }^{23}$, l'émergence d'une «théorie critique de la musique » est manifeste dans les écrits récents sur la théorie schenkérienne ${ }^{24}$. Je fais référence ici à trois contributions du Britannique Nicholas Cook de même qu'à trois contributions de Richard Cohn ${ }^{25}$, tous deux des théoriciens sympathiques aux idées de Schenker.

Je présenterai un résumé de ces contributions en gardant à l'esprit deux objectifs bien précis ${ }^{26}$. Premièrement, il s'agit de relever les propositions élaborées par Cook et Cohn qui constituent une solide amorce de lecture critique de l'appropriation des idées de Schenker par les théoriciens-analystes anglo-américains, de « l'américanisation ${ }^{27}$ de Schenker, et de démontrer que

23 On pense par exemple à une étude qui serait entreprise par un théoricien de la musique en association avec un anthropologue ou un sociologue, et dont le but serait d'élaborer un cadre théorique permettant d'analyser le statut idéologique et les politiques constitutives du discours dominant dans la discipline de la théorie musicale, en positionnant ce discours, son rôle et son impact dans le processus culturel global, pratique courante, par exemple, chez les ethnomusicologues.

24 À titre d'efforts dans cette direction, on note la tenue en mars 1994 d'un congrès intitulé « Critical Perspectives on Schenker: Toward a New Research Paradigm » au Center for Continuing Education, University of Notre Dame, Indiana, réunissant entre autres Allen Keiler, David Neumeyer et Pieter C. van den Toorn. Voir aussi Richard Littlefield et David Neumeyer, « Rewriting Schenker : Narrative - History - Ideology », Music Theory Spectrum 14, ${ }^{\circ} 1$ (1992) : 38-65 ; Lawrence Kramer « Haydn's Chaos, Schenker's Order ; or, Hermeneutics and Musical Analysis : Can They Mix ?», 19th Century-Music 16, no 1 (1992) : 3-17 (Kramer propose une réconciliation de l'entreprise de l'analyse musicale, vue ici dans une perspective schenkérienne, et de la critique herméneutique) ; dans la même revue, voir aussi Scott Burnham et Lawrence Kramer, «The Criticism of Analysis and the Analysis of Criticism ; Criticizing, Analyzing Analysis », 70-76; 76-79.

25 La première des contributions présentées ici est le résultat d'une collaboration entre Richard Cohn et Douglas Dempster.

26 Pour des raisons évidentes d'espace, je ne commenterai pas l'ensemble des arguments respectifs de Cook et de Cohn. J'invite toutefois le lecteur à consulter les articles mentionnés pour une perspective complète sur les hypothèses qui y sont élaborées.

27 Pour un historique de la dissémination des idées de Schenker aux États-Unis et en Europe, voir David Beach, "The Current State of Schenkerian Research », Acta musicologica 57 (1985): 275-99 ; et William Rothstein, « The Americanization of Heinrich Schenker », paru initialement dans In Theory Only 9, n 1 (1986) : 5-17, et repris dans Schenker Studies, éd. par Hedi Siegel 
ce qui est ciblé dans leurs propos est la volonté, à la fois implicite et explicite des schenkériens contemporains, d' "élever » la théorie schenkérienne au rang de discours scientifique, de l'ériger en système infaillible, tel un passeport permettant l'accès à un univers de vérité absolue pour qui réussit à naviguer dans les méandres de l' «épistémè » américaine. Deuxièmement, il s'agit d'alerter le lecteur au fait qu'une révision critique du discours schenkérien contemporain doit prendre en compte des phénomènes de médiation d'une telle complexité qu'elle se doit de faire appel à une stratégie interprétative capable de réconcilier plusieurs points de vue et, ultimement, des versions contradictoires d'un même phénomène.

Une approche critique du discours schenkérien par des théoriciens qui cherchent à débarrasser les idées de Schenker de l'universalité étriquée dont elles ont été affublées dans les dernières décennies plutôt qu'à les évacuer des débats qui agitent à l'heure actuelle la communauté des musicologues et, à un degré moindre, des théoriciens, mérite toute notre attention dans le contexte d'une révision en profondeur des discours contemporains dominants en musicologie et en théorie de la musique. Cette approche critique ne peut être que bienvenue pour quiconque reconnaît d'emblée que la théorie de Schenker permet de sonder les chefs-d'œuvre du répertoire tonal comme nulle autre théorie ne l'a fait avant elle.

\section{Trois contributions de Nicholas Cook}

Dans sa première contribution, intitulée "Schenker's Theory of Music as Ethics ${ }^{28}$, Cook examine le contexte philosophique de la Vienne des années 1890-1930 afin de comprendre la nature polémique et quasi-philosophique des écrits de Schenker, aspect de son œuvre qui a pour ainsi dire été totalement évacué par les praticiens contemporains de l'analyse schenkérienne. Il retrace l'influence du critique viennois Eduard Hanslick, du philosophe Arthur Schopenhauer et de la tradition idéaliste allemande relative à la pensée de Schenker. Puis, par le biais d'une lecture critique de l'article de Schenker «Weg mit dem Phrasierungsbogen ${ }^{29}$, Cook examine comment le très influent

(Cambridge : Cambridge University Press, 1990), 193-203. Pour une lecture critique de la dissémination des idées de Schenker aux États-Unis, voir entre autres Joseph Kerman, Musicology, 60-112.

28 The Journal of Musicology 7, $\mathrm{n}^{\circ} 4$ (1989) : 415-39.

29 Paru dansDas Meisterwerk in der Musik (Jahrbuch I) en 1925 (p. 41-60); traduction sous le titre de « Let's do away with the phrasing slur » dans Sylvan Kalib, « Thirteen Essays from the Three Yearbooks Das Meisterwerk in der Musik by Heinrich Schenker : An Annotated Translation » (thèse de doctorat, Northwestern University, 1973), 1:52-83. 
critique viennois Karl Kraus, ou plus précisément sa conception éthique de l'art et de l'artiste, a contribué à façonner les idées maîtresses de la philosophie de Schenker. Ce faisant, Cook sonde une dimension de l'œuvre de Schenker qui, il y a peu de temps, était encore occultée, comme nous le rappelle son commentaire d'ouverture : "Jusqu'à dernièrement, les éditions publiées des écrits de Schenker mettaient en application [une] approche sélective, ou même soumise à la censure, en ce qu'elle allait jusqu'à supprimer de l'original des passages considérés inutiles ou indésirables ${ }^{30}$.»

Dans la dernière partie de son article, Cook affirme que la théorie schenkérienne constitue "une application dans le domaine de la musique de la position éthique générale incarnée par la vie et l'œuvre de Kraus. » Mais, ajoute-t-il, « ce n'est pas là la manière dont nous lisons Schenker à l'heure actuelle ; [...] Comment pouvons-nous justifier un usage aussi sélectif de l'œuvre de Schenker ${ }^{31}$ ? » En réponse à cette question, Cook identifie certaines des prémisses constitutives de la pensée de Schenker qui ont été évacuées lors de l'appropriation de ses idées par l'école américaine, plus précisément (1) le lien entre les principes structurels des chefs-d'œuvre et la nature ; et (2) la notion de génie qui, comme chacun le sait, est profondément enracinée dans son œuvre. Puis, il démontre que l'élimination de ces prémisses ne constitue pas une simple extension des idées originales de la théorie en mettant en évidence les conséquences d'une telle interprétation lacunaire sur l'intégrité des idées de Schenker.

Cook avance qu'une explication possible de l'usage contemporain de la théorie schenkérienne réside dans le fait que plusieurs la conçoivent en tant que théorie de la perception, ce qui constitue en soi une fausse prémisse, argument qu'il reprendra et développera plus avant dans sa deuxième contribution. Cook termine ce premier tour de piste en proposant qu' « une analyse schenkérienne ne décrit pas en principe comment une œuvre est entendue; elle indique plutôt comment s'en faire une certaine image mentale, ou comment l'écouter d'une

30 « Until recently, published editions of Schenker's writings embodied this selective, or even censorious, approach to the extent of actually deleting passages of the original that were considered unnecessary or undesirable. » Cook, « Schenker's Theory of Music as Ethics », 415. En dépit des recherches d'un chercheur comme William Pastille, par exemple, qui visent spécifiquement à démontrer les relations entre la théorie de Schenker et la tradition idéaliste allemande, cet aspect de la théorie de Schenker n'a pas été retenu par les praticiens de l'analyse schenkérienne. Voir entre autres, «Ursatz: The Musical Philosophy of Heinrich Schenker » (thèse de doctorat, Cornell University, 1985), et « Heinrich Schenker, Anti-Organicist », 19thCentury Music 8, $\mathrm{n}^{\circ} 1$ (1984) : 29-36.

31 « an application within the field of music of the general ethic position epitomized in Kraus' life and work. " "But that is not how we read Schenker today ; $[\ldots]$ How can we justify using Schenker's work in so selective a manner? » Ibid., 433. 
manière créatrice ${ }^{32}$. » Une telle observation laisse croire à un désir de réinvestir le champ du ludique, d'établir un espace au sein de l'entreprise théorique où l'esprit de jeu et l'intrusion de l'imaginaire trouveraient droit de cité. Cook conclut en proposant, à l'instar de Christopher Lewis ${ }^{33}$, de remplacer par la métaphore le critère de validation et de vérifiabilité d'une analyse en vigueur dans le discours théorique actuel, soit les procédures explicatives propres aux sciences.

Comme toute autre métaphore, l'analyse schenkérienne peut être pratiquée de manière satisfaisante ou insatisfaisante, convaincante ou non convaincante. Mais, tel qu'observé par Lewis, aucune explication métaphorique ne peut être vraie ou fausse dans un sens absolu ou scientifique ; au contraire des théories scientifiques incompatibles, des analyses contradictoires d'une même pièce de musique peuvent être également valides [...] Il ne s'agit pas de savoir quelle approche analytique est la plus valide ou la plus correcte au sens abstrait mais bien de savoir ce qu'on attend d'une analyse ${ }^{34}$.

À mon avis, l'aspect le plus important de cette première contribution réside dans ce début d'interprétation d'un aspect jusque-là censuré de l'œuvre de Schenker: la nature polémique et quasi-philosophique de ses écrits. En proposant une lecture des idées de Schenker en tant que phénomènes conditionnés historiquement, Cook met aussi en évidence comment l'évacuation de cette dimension historique a contribué à l'élaboration d'une interprétation lacunaire de la théorie. Ce faisant, il souligne l'urgence d'une évaluation critique du processus d'appropriation des idées de Schenker par les théoriciens-analystes anglo-américains.

La deuxième contribution de Cook, "Music Theory and "Good Comparison" : A Viennese Perspective ${ }^{35}$, s'inscrit dans le débat sur l'image

32 " A schenkerian analysis is not primarily a description of how a piece is, in fact, heard ; it is rather a prescription for imagining it in a certain manner, or hearing imaginatively. » Ibid., 436.

33 " Mirrors and Metaphors : Reflections on Schoenberg and Nineteenth-Century Tonality ", 19thCentury Music 11, $\mathrm{n}^{\circ} 1$ (1987) : 26-42.

34 "Like any other metaphor, Schenkerian analysis can be applied in a satisfying or unsatisfying manner, convincingly or unconvincingly. But as Lewis observes, no metaphorical explanation can be true or false in an absolute or scientific sense ; unlike conflicting scientific theories, contradictory analyses of a given piece of music can be equally valid [...] This is not a question of which analytical approach is the more valid or correct in the abstract sense ; it is a question of what the analysis is wanted for. " Ibid., 437.

35 Journal of Music Theory 33, $\mathrm{n}^{\circ} 1$ (1989) : 117-41. 
scientifique de la théorie de la musique mentionné ci-haut (voir note 17), et est donc conçue en réponse aux prises de position des théoriciens Brown et Dempster. Le propos de cet article est d'établir ce qui constitue la validité d'une analyse. Pour ce faire, Cook reprend les éléments exposés dans la première contribution. Sans entrer dans les méandres de son argumentation, notons que, dans un premier temps, il cherche à démontrer que la prémisse selon laquelle la théorie schenkérienne est une théorie de la perception - explicite dans les travaux de Lerdahl et Jackdendoff ${ }^{36}$, de Narmour et de Keiler $^{37}$ - est en soi une fausse prémisse, en d'autres termes, que celle-ci ne fait pas partie des fondements de la pensée de Schenker. Dans un deuxième temps, Cook démontre comment le projet de théorie scientifique de Benjamin Boretz et de Milton Babbitt ${ }^{38}$ résulte en une théorie des partitions musicales, au même titre que pour Schönberg et, plus spécifiquement, Schenker, pour qui « la partition n'est pas une représentation de la musique ; elle est la musique ${ }^{39}$, interprétation fondée sur la lecture de l'article «Weg mit dem Phrasierungsbogen » déjà mentionné. Ce faisant, Cook met en évidence le paradoxe inhérent à cette situation, soit : une théorie qui, tout en prétendant à la rigueur scientifique, est fondée sur une conception de la partition musicale « qui relève de la métaphysique propre au XIX ${ }^{\mathrm{e}}$ siècle plutôt que de la psychologie propre au $\mathrm{XX}^{\mathrm{e}}$ siècle ${ }^{40}$. » Le prix à payer pour résoudre ces problèmes, affirme Cook, est «l'abandon de l'image d'une théorie scientifique de la musique ${ }^{41}$. »

En troisième lieu, Cook développe plus avant l'idée de la substitution de la métaphore au modèle scientifique comme critère de validation d'une analyse, les objets de la comparaison étant ici les espèces fuxiennes et la surface de la musique. Finalement, il prend position dans la controverse « universalisme contre particularisme » soulevée par Brown et Dempster en démontrant comment Schenker est à la fois un universaliste, par la généralisation inhérente au processus de réduction de l'œuvre jusqu'à sa structure profonde (Ursatz), et un particulariste. Pour lui, la disparition progressive des éléments constituants de la surface de la musique dans le processus de réduction ne diminue en rien leur signification pour l'ensemble de l'œuvre, mais indique simplement

36 Ibid., 118-19.

37 Ibid., 120, 129-30.

38 lbid., 120-23.

39 " the score is not a representation of the music ; it is the music. " Ibid., 122.

40 « a conception of the scores that makes more sense in terms of nineteenth-century metaphysics than twentieth-century psychology. » Ibid., 121-22.

41 « dispensing with the image of a scientific theory. " Ibid., 123. 
«qu'ils n'assument pas un rôle structurel en termes fonctionnels du point de .vue de la tonalitét ${ }^{42}$.»

Cook avance que la valeur d'une analyse schenkérienne ne réside pas tant dans une concordance parfaite entre la représentation analytique et l'œuvre que dans la capacité à mettre en lumière "les divergences entre le design de la surface et la structure sous-jacente", à révéler "comment la musique est animée par la tension entre structure de surface et structure profonde, le tout et les parties ${ }^{43}$. » Cook accompagne ce commentaire d'un bref éclaircissement : «L'analyse schenkérienne telle qu'elle se pratique actuellement, il me semble, tend à accorder trop d'importance à l'unité per se, plutôt qu'aux relations dynamiques entre les différents niveaux ${ }^{44}$."

Cook termine en précisant que le cadre qu'il propose pour valider une théorie musicale et la pratique analytique ne prétend pas, comme la connaissance scientifique, à la vérifiabilité, à la preuve hors de tout doute, mais plutôt à «illuminer la surface ${ }^{45}$, dans les mots de Judith et A. L. Becker, «à comprendre et non pas expliquer ${ }^{46}$. De tels propos, situés en quelque sorte aux antipodes de la rhétorique formaliste, surprennent lorsque l'on songe à ce que l'intrusion d'une référence poétique - ici le mot « illuminer » est utilisé dans le sens d'expliquer - représente pour quiconque est familier avec le jargon académique des théoriciens anglo-américains. En effet, la référence poétique ou l'envolée quelque peu lyrique sont non seulement suspectes mais en général synonymes de subjectivité déplacée quand ce n'est pas d'indigence intellectuelle.

Cette deuxième contribution circonscrit de plus en plus nettement la cible visée. Cook utilise le « nous ", s'incluant ainsi naturellement dans la communauté des théoriciens-analystes anglo-américains; il utilise ce ton décontracté de l'échange civilisé qui est le sien; enfin, il centre son propos surtout sur les auteurs qui ont critiqué Schenker (Narmour, Keiler et Kerman) ou encore proposé des adaptations et des extensions à sa théorie (Lerdahl et Jackendoff),

42 «they do not have a structural role in tonal-functional terms » Ibid., 132. Cook répond ainsi à la critique la plus fréquemment formulée à l'endroit de Schenker par les musicologues, notamment par Joseph Kerman qui est ici pris à parti, voir p. 131-32.

43 « reveals the divergence between surface design and underlying structure ; [...] how the music is animated by the tension between foreground and background, whole and parts. » Ibid., 132.

44 "Present-day Schenkerian analysis, it seems to me, tends to put too much emphasis on unity per $s e$, rather than on the dynamic relationships between different levels. "Ibid., 141.

45 «illuminates the surface. »Ibid., 133. Le lecteur pourra consulter Music, Imagination, and Culture (Oxford : Clarendon Press, 1990), où Cook poursuit sa réflexion sur le sujet.

46 « to understand, not to explain. » Ibid., 135. Citation tirée de « Responses to Feld and Roseman, » Ethnomusicology 28, no 3 (1984) : 454-56; 455. 
plutôt que sur les adhérents sinon inconditionnels à tout le moins très peu enclins à la critique. Toutefois, ces précautions oratoires n'obscurcissent pas pour autant le but du propos, qui consiste à mettre en cause l'impératif de la rectitude, de l'infaillibilité d'une analyse et d'une théorie en tant que preuve de leur valeur intrinsèque. Il s'agit d'un impératif dont les origines remontent aux prises de position des théoriciens de Princeton dans les années 1960-70 et de Milton Babbitt en particulier. Ce dernier, un partisan du formalisme à tout crin, a été le principal instigateur, à l'époque, de cette tendance à la systématisation et, conséquemment, à l'uniformisation, au nivellement, à la standardisation du discours théorique en général et, cela va de soi, du discours schenkérien ${ }^{47}$. Les critiques formulées par Cook dans cette deuxième contribution montrent quelques-unes des grandes orientations possibles dans l'élaboration d'une lecture critique du discours schenkérien contemporain. On observera toutefois qu'il ne met pas en question le statut idéologique de la pratique des théoriciensanalystes ou les structures institutionnelles qui la façonnent. Ce sera en quelque sorte le propos de sa troisième contribution.

En effet, dans le cadre de l'enquête sur le devenir de la théorie mentionnée ci-haut (voir note 18), Cook, faisant référence à une publication antérieure, rappelle avoir interprété Schenker en apologiste de Hanslick ${ }^{48}$, fervent défenseur de l'idéologie de l'autonomie de la musique, et affirme qu'il en va de même pour les Forte, Lerdahl et Jackendoff et même Nattiez ${ }^{49}$. Il poursuit sa réflexion en proposant le raisonnement suivant : si la théorie de la musique, dans sa forme actuelle, a pour objet d'étude une structure autonome (sans relation avec les contextes sociaux, politiques, économiques, etc.), et si l'idéologie de l'autonomie est précisément l'objet de la critique déconstructionniste, «ce que j'appelle une théorie critique de la musique, alors, érode l'identité même de la théorie de la musique en tant que discipline. »Il ajoute : "elle porte la promesse, plutôt, d'une musicologie qui est conçue plus généreusement que l'étroite abstraction de la caricature de Kerman. » Puis, il précise :

Ce que j'entends par ceci est une musicologie qui est critique au sens où elle est consciente de son propre statut idéologique, où elle postule son

47 Voir entre autres le compte rendu par Babbitt de Structural Hearing de Felix Salzer, dans Journal of the American Musicological Society 5, $\mathrm{n}^{\circ} 2$ (1952) : 260-65. «Contemporary Music Composition and Music Theory as Contemporary Intellectual History », dans Barry S. Cook et al., Perspectives in Musicology (New York : W. W. Norton, 1972), 151-84. « Past and Present Concepts of the Nature and the Limits of Music » et « The Structure and Function of Musical Theory », éd. par Benjamin Boretz et Edward T. Cone, Perspectives on Contemporary Music Theory (New York: W. W. Norton, 1972), 3-9, 10-21.

48 Cook, "Schenker's Theory of Music as Ethics », 426.

49 Cook, « The Future of Theory », 71. 
propre rôle dans le processus culturel [...] La plupart des analyses schenkériennes publiées (et plus encore non publiées) sont fondées sur l'hypothèse que le but visé est de montrer comment les objets musicaux sont unifiés. Nous n'avons pas tendance à percevoir cette attitude comme étant motivée idéologiquement [...] L'américanisation de Schenker (pour reprendre le terme de Rothstein ${ }^{[50]}$ ) a entraîné l'oubli des origines polémiques de l'idée de l'unité musicale, dans les controverses, aux $\mathrm{XVIII}^{\mathrm{e}}$ et XIX ${ }^{\mathrm{e}}$ siècles, sur l'émancipation de la musique instrumentale et sur la question du classicisme contre romantisme, tout comme elle a entraîné la suppression des aspects politiques et éthiques de la Weltanschauung de Schenker. La recherche historique sur les sources de la théorie schenkérienne [...] peut bien ne pas changer la façon dont nous employons les techniques schenkériennes, mais elle peut nous aider à acquérir une conscience critique du bagage intellectuel qui leur sont inhérentes - et peut-être aussi des motifs esthétiques et des structures institutionnelles qui nous incitent en premier lieu à les utiliser ${ }^{51}$.

Cook termine en proposant une solution au problème de fixation des schenkériens sur l'unité de l'œuvre. Il suggère qu'en dépit du fait que l'analyse schenkérienne soit « fondée sur le concept d'unité [...], elle traite de tension, de conflit, d'absence d'unité ${ }^{52}$. » Cette proposition, sur laquelle je reviendrai dans le contexte de la troisième contribution de Richard Cohn, cible à nouveau l'aspect de l'appropriation des idées de Schenker par les théoriciens-analystes

50 Voir Rothstein, «The Americanization of Heinrich Schenker ».

51 «What I am calling critical music theory, them, erodes the very identity of music theory as a discipline. It holds out the promise, rather, of a musicology that is more generously conceived than the narrow abstraction of Kerman's caricature. " « What I mean by this is a musicology that is critical in the sense of being aware of its own ideological status, of positing its own role in the cultural process [...] Much published (and even more unpublished) Schenkerian analysis works on the assumption that the aim is to demonstrate the manner in which musical objects are unified. We don't tend to perceive this as an ideologically motivated aim ; [...] The Americanization of Schenker (to use Rothstein's term) involved forgetting the polemical origins of the idea of musical unity, in 18th- and 19th-century controversies over the emancipation of instrumental music and classicism vs. romanticism, just as it involved suppressing the political and ethical aspects of Schenker's Weltanschauung. Historical research into the sources of Schenkerian theory [...] may not change the way we employ Schenkerian techniques, but it can help give us a critical awareness of the intellectual baggage that comes with them - and maybe also of the aesthetic motives and institutional structures that lead us to employ them in the first place." Cook, «The Future of Theory », 71.

52 « [Schenkerian analysis] is predicated on the concept of unity [...], but about tension, conflict, disunity », Ibid., 71. Cook propose cette solution en réponse à la communication «Three 
anglo-américains qui est le plus vivement attaqué dans les débats entre les musicologues anglo-américains à l'heure actuelle : soit que la démonstration de l'unité et de la cohérence des œuvres entraîne avec elle la prétention à l'universalité et à l'infaillibilité de la théorie schenkérienne.

J'ai choisi de présenter un condensé de ces trois contributions de Cook non seulement parce qu'elles constituent des jalons importants dans l'élaboration d'une révision critique du discours schenkérien contemporain, mais aussi parce que deux d'entre elles ont été élaborées en tant que prises de position dans le cadre des remises en question amorcées dernièrement par certains théoriciensanalystes. Les critiques formulées par Cook, son interprétation des problèmes auxquels fait face la discipline et les solutions qu'il propose prêtent naturellement le flanc à la critique, comme en témoigne la réponse de Douglas et Dempster ${ }^{53}$. Étant donné leur position dans le débat, position qui favorise un discours théorique dont les procédures explicatives s'inspirent de la science, il n'est pas surprenant que ces derniers réagissent surtout à la suggestion de Cook de substituer la métaphore aux procédures explicatives de nature scientifique ${ }^{54}$. Richard Cohn poussera plus avant la critique de certaines des assertions de Cook, comme nous le verrons dans sa lecture critique de l'appropriation des idées de Schenker dont il s'agira maintenant d'exposer les principaux éléments.

\section{Trois contributions de Richard Cohn}

Dans la première contribution, « Hierarchical Unity, Plural Unities : Toward a Reconciliation ${ }^{55}$, Cohn et Dempster remettent en question la validité du canon de l'unité et de la cohérence dans la pratique schenkérienne - canon étant compris ici dans son acception traditionnelle qui provient du grec kanôn, soit règle, norme, loi. Les auteurs examinent la prémisse de ce canon selon laquelle "l'unité musicale se trouve non pas "exposée sur" la "surface" complexe [...] d'une composition mais plutôt " cachée dans "quelque simplicité structurelle "sous-jacente" et, plus spécifiquement, que «la structure sousjacente qui unifie la complexité de la musique telle que reflétée par la surface est hiérarchique ${ }^{56}$.»

Challenges to the Schenkerian View of Motive », présentée par Richard Cohn au colloque Music Theory Canada (London, Ontario, mars 1990), laquelle est à l'origine des trois contributions qui seront discutées ci-dessous.

53 Matthew Brown et Douglas J. Dempster, «The Scientific Image of Music Theory ».

54 Ibid., 257-60.

55 Richard Cohn et Douglas Dempster, dans Disciplining Music : Musicology and Its Canons, 15681. 
Après avoir défini rigoureusement le concept de hiérarchie et établi qu'une interprétation adéquate du concept de prolongation implique la nature hiérarchique des modèles schenkériens de conduite des voix, les auteurs procèdent à une interprétation du concept de hiérarchie tel que présenté dans les écrits et dans la pratique analytique des Schachter, Rothgeb, Burkhart et Rothstein. Cette interprétation révèle des prises de position qui «mettent en question, implicitement, le principe schenkérien central voulant que la structure fondamentale soit la seule source de la structure organique et de l'unité compositionnelle, et, conséquemment, la conception hiérarchique des compositions $^{57}$. » Cohn et Dempster concluent à l'absence de consensus au sein de la communauté schenkérienne sur la relation existant entre la structure prolongationnelle (le modèle incluant principalement l'harmonie, la mélodie et le contrepoint) et les caractéristiques extra-prolongationnelles d'une œuvre («durée des évènements et leur position métrique ; [...] texture, densité et timbre $;[\ldots]$ patterns de figuration de la surface et relations motiviques $;[\ldots]$ conventions de forme et de topos ; [...] mise en musique d'un texte ${ }^{58}$.»)

De même, une interprétation de la conception schenkérienne du parallélisme motivique énoncée par Schenker et reprise dans les écrits d'auteurs comme Schachter, Rothgeb, Burkhart et Beach révèle l'existence d'une faille entre l'énoncé des principes théoriques et la pratique analytique. Celle-ci s'accomode d'une plus grande flexibilité que ne le prescrit la définition originale dans le but ultime de produire des analyses d'une plus grande richesse. En conclusion, les auteurs présentent une alternative à la conception de l'unité musicale, soit le concept de product networks ${ }^{59}$ qui réconcilie le canon de l'unité engendrée hiérarchiquement et "un deuxième canon indépendant », dénommé canon de la richesse ${ }^{60}$, dont la principale caractéristique est d'offrir la flexibilité nécessaire à la production d'analyses intéressantes.

56 " musical unity is to be found not " exposed on " the complex [...] " surface " of a composition, but rather " hidden in " some " underlying " structural simplicity "; " the underlying structure that unifies the surface complexity of music is hierarchical ». Ibid., 156.

57 « implicitly represent a challenge to the central tenet that the fundamental structure is the sole source of organic structure and compositional unity, and thus to the hierarchical view of compositions. "Ibid., 166.

58 « the duration of events and their metric position ; [...] texture, density, and timbre $;[\ldots]$ patterns of surface figuration and motivic relationships $;[\ldots]$ conventions of form and topos $;[\ldots]$ the setting of texts. » Ibid., 164.

59 Le concept de product network a été présenté à l'origine par David Lewin dans Generalized Musical Intervals and Transformations (New Haven : Yale University Press, 1987). Le lecteur désireux d'en savoir plus sur l'adaptation du concept de product network par Cohn et Dempster pourra consulter «Alternative Conceptions of Musical Unity : Some Speculations » aux pages 170-78 de l'article à l'étude ici. 
Dans sa deuxième contribution, « The Autonomy of Motives in Schenkerian Accounts of Tonal Music $»^{61}$, Cohn s'applique à démontrer que la conception schenkérienne standard de la relation entre motif et structure ne rend pas compte de la pratique analytique de la plupart des schenkériens. Dans un permier temps, il retrace l'évolution graduelle de la définition de l'unité dans la pensée de Schenker dans les écrits des années 1890 à 1930, où l'Ursatz devient progressivement la source unique de toute unité compositionnelle, « conception de l'unité qui continue à être maintenue par les interprètes modernes des théories de Schenker ${ }^{62}$. » Cohn examine aussi la conception schenkérienne du motif ou, plus spécifiquement, la transformation graduelle de la conception de son rôle en tant que force organisationnelle. Il conclut que le motif, progressivement redéfini par Schenker, sera finalement conçu, au même titre que les autres paramètres de l'œuvre, en fonction de sa « dérivabilité de l'Ursatz au moyen des transformations de la conduite des voix ${ }^{63}$. »

Dans un deuxième temps, Cohn reconnaît que les prises de position de Rothgeb, de Schachter et de Beach sur le rôle du motif dans la séquence décisionnelle qui préside aux choix analytiques découlent directement des principes théoriques énoncés par Schenker, principes qu'il résume ainsi : « $\mathrm{La}$ reconnaissance de relations motiviques résulte de l'analyse mais n'influence pas le processus analytique ${ }^{64}$.» Puis, passant habilement le scalpel dans certains choix analytiques opérés par les schenkériens mentionnés plus haut, il découvre qu'ils adoptent exactement la stratégie contraire, c'est-à-dire qu'ils « considèrent fréquemment l'information motivique en tant que guide dans le processus de construction d'un graphe ${ }^{65}$. » Selon Cohn, quelles que soient les motivations qui sous-tendent l'adoption de cette stratégie, toutes mettent en évidence différents types d'incompatibilités entre les principes théoriques et la pratique analytique.

$60 \mathrm{Ibid} ., 178$.

61 Music Theory Spectrum 14, $\mathrm{n}^{\circ} 2$ (1992) : 150-70.

62 « view of unity which continues to be maintained by modern interpreters of Schenker's theories. " Ibid., 152.

63 « its derivability from the Ursatz via voice-leading transformations. » Ibid., 153.

64 « Recognition of motivic relations results from analysis, but does not influence the analytic process. » Ibid., 155.

65 « frequently look to motivic information as a guide to a process of constructing graphs. » Ibid., 156. Tel qu'observé par Cohn, cette apparente contradiction se retrouve par exemple tout autant chez Edward Laufer dans sa recension de la traduction anglaise de Der freie Satz de Schenker, publiée dans Music Theory Spectrum 3 (1981) : 166-67, que chez Ernst Oster dans « Re : A New Concept of Tonality (?) », Journal of Music Theory $4, \mathrm{n}^{\circ} 1$ (1960): 88. On notera que le matériel de cette partie de l'article de Cohn est dérivé de la contribution précédente, « Hierarchical Unity, Plural Unities », produite en collaboration avec Douglas Dempster. 
Dans un troisième temps, par le biais d'une interprétation critique de certaines analyses de Schenker, Cohn démontre que la pratique analytique de ce dernier «n'est pas toujours fondée sur ses [propres] théories de ce qui constitue une pratique analytique acceptable ${ }^{66}$. » Puis - et ceci est crucial pour interpréter l'appropriation des idées de Schenker par les théoriciens-analystes anglo-américains - Cohn observe que cet état de fait a perturbé la discipline vers la fin des années 60 ; en effet, les schenkériens ont assisté à l'émergence de deux camps à la suite d'un échange entre John Rothgeb et Carl Schachter au sujet d'un Moment musical de Schubert ${ }^{67}$ : les tenants de la stricte fidélité aux écrits théoriques de Schenker, et les tenants d'une attitude plus flexible inspirée de la pratique même de Schenker l'analyste.

Les lignes de combat ont été clairement établies lors de cet échange : Rothgeb maintenant le standard des écrits théoriques de Schenker, Schachter la flexibilité et la richesse de sa pratique analytique. Mais aucune scission ne s'est matérialisée. Les partisans ont simplement cessé le dialogue, accordant évidemment plus de valeur à l'unité communautaire qu'à la prise de conscience communautaire. Néanmoins, parce qu'il était très réel, le problème représenté par cet échange n'est pas disparu ; au lieu de cela, il a pénétré le travail des chercheurs individuels qui ont du élaborer des positions personnelles sans le bénéfice d'un dialogue public. Certains ont mis au point des positions cohérentes et les ont maintenues, tacitement, dans tous leurs travaux ; d'autres continuent à élaborer une position en relation à l'ensemble des options disponibles; d'autres encore utilisent la théorie de Schenker comme modèle pour évaluer le travail des autres, mais cherchent dans ses analyses les paradigmes à partir desquels modeler leur propre pratique analytique, peu soucieux du danger qu'il y a à rendre les autres responsables d'un standard qu'on ne maintient pas soi-même ${ }^{68}$.

À la quatrième étape de sa démonstration, Cohn établit comment l'existence, dans la pratique analytique, de deux types de génération du motif remet en

66 « is ńot always based on his theories about what constitutes acceptable analytic practice. » Cohn, "The Autonomy of Motives in Schenkerian Accounts of Tonal Music », 162.

67 Carl Schachter, « Schubert, Op. 94 No. 1 : A Schenkerian Analysis »; John Rothgeb, « Another View of Schubert's Op. 94 No. 1 »; Carl Schachter, « More About Schubert's Op. 94 No. 1 ». Ces trois articles sont parus dans le Journal of Music Theory $12, \mathrm{n}^{\text {os }} 1$ et 2 (1968) : 222-39; et 13, $\mathrm{n}^{\mathrm{os}} 1-2$ (1969) : 128-39, 218-29. Ils ont été réimprimés dans Readings in Schenker Analysis, éd. par Maury Yeston (New Haven : Yale University Press, 1977), 171-201.

68 "With this exchange, the battle lines were clearly drawn, with Rothgeb upholding the standard of Schenker's theoretical writings, Schachter the flexibility and richness of his analytic practice. 
question le statut de l'Ursatz en tant que seule source génératrice d'unité. Il pose en effet la question cruciale : "Si toutes les entités sont générées uniquement à partir de l'Ursatz, comment certaines entités peuvent-elles aussi être générées à partir des plus petites diminutions ${ }^{69}$ ? » et conclut en affirmant « si l'Ursatz n'est pas la seule source de l'unité motivique, alors l'Ursatz n'est pas la seule source de l'unité compositionnelle ${ }^{70}$."

Dans la section finale, Cohn propose trois solutions potentielles aux incompatibilités entre théorie et pratique relevées dans sa réflexion. Elles représentent, chacune à sa manière, une attitude propre aux théoriciensanalystes anglo-américains et méritent donc notre attention. La première solution consiste à nier la nécessité de relier la théorie à la pratique, solution que Cohn rejette en utilisant les trois arguments suivants :

(1) La parfaite adéquation entre théorie et pratique est en effet perçue par certains comme étant indésirable parce qu'elle privilégie une rigidité excessive plutôt que les ressources de l'intuition, et ce au détriment de la fécondité de la théorie, de sa capacité à produire des résultats intéressants. D'une part, Cohn souligne que la théorie schenkérienne est issue d'une tradition intellectuelle "qui n'évaluait pas les théories uniquement selon leur degré de confirmation empirique, mais aussi selon leur capacité à incarner des idéaux spirituels universels $^{71} »$. Il rejoint ici Nicholas Cook, dont la première contribution propose une lecture des idées de Schenker en tant que phénomènes conditionnés historiquement. Il souligne aussi que, même si « Schenker rejetait l'attitude scientifique avec véhémence », « il ne rejetait absolument pas la précision dans

\footnotetext{
But no fissure materialized. Evidently placing a higher value on communal unity than on communal self-awareness, the partisans simply ceased dialogue. Nonetheless, because it was a very real one, the issue represented by this exchange did not disappear ; instead, it submerged into the work of individual scholars, who were left to work out private positions without benefit of public dialogue. Some have crafted consistent positions and maintained them tacitly throughout their work ; others continue to evolve a position along the spectrum of available options ; still others use Schenker's theory as a model for evaluating the work of others, but look to Schenker's analyses for paradigms on which to model their own analytic practice, heedless of the hazards of holding others to a standard that one does not oneself maintain. » Cohn, « The Autonomy of Motives in Schenkerian Accounts of Tonal Music », 162-63.

69 « If all entities are generated only from the Ursatz, how can some entities also be generated from their tiniest diminutions? » Ibid., 166.

70 « if the Ursatz is not the sole source of motivic unity, then the Ursatz is not the sole source of compositional unity. » Ibid., 167.

71 « which evaluated theories not only according to their degree of empirical confirmation, but also to the extent to which they embodied universal spiritual ideals. » Ibid., 167.
} 
la formulation et la présentation des résultats analytiques, ni la cohérence et la rigueur dans leur évaluation ${ }^{72}$. $\gg$ Il conclut en commentant la situation qui prévaut au sein de la discipline: "Il est ironique de constater que si les schenkériens veulent à la fois maintenir les principes musico-théoriques et les résultats analytiques de Schenker, ils sont forcés de rejeter sa métathéorie ${ }^{73}$. »

(2) Cohn relève une deuxième source de problème émanant de cette première solution :

Les schenkériens ont été prompts à critiquer les chercheurs dont la pratique analytique n'est pas conforme aux théories de Schenker. En désavouant la nécessité de conformer leur propre pratique aux théories de Schenker, ils s'accordent une licence qu'ils refusent aux autres ${ }^{74}$.

(3) Enfin, le rejet d'une parfaite adéquation entre théorie et pratique soulève selon Cohn la question de la définition de la pratique analytique. L'auteur suggère dans un premier temps que cette pratique doit inéluctablement être fondée sur un quelconque ensemble de principes généraux témoignant d'une certaine forme d'organisation; dans un deuxième temps, il ajoute que tenter d'expliquer la pratique analytique en ayant recours à une quelconque forme "d'intuition musicale guidée par une longue expérience » conduit $(a)$ au mieux à ce que les schenkériens concèdent « que le système de connaissances sousjacentes qui aiguillonne leurs jugements dans des cas particuliers est structuré, et que les principes qui structurent ce système sont partiellement capables d'organisation rationnelle », et $(b)$ au pire à ce que ceux qui ont subi les attaques des schenkériens ou encore les néophytes sceptiques réduisent cette « intuition inaccessible » à « un écran pour un ensemble de jugements arbitraires et non organisés, fondés plus sur le népotisme que sur le mérite ${ }^{75}$.»

72 «the scientific attitude which Schenker vehemently rejected [...] ; « he most emphatically did not reject precision in the formulation and presentation of analytic results, nor consistency and rigor in their evaluation. » Ibid., 167-68.

73 "The irony is that, if Schenkerians want to maintain both Schenker's music-theoretical principles and his analytic results, they are compelled to reject his metatheory. " Ibid., 168.

74 "Schenkerians have been quick to criticize other scholars whose analytic practice fails to conform to Schenker's theories. By denying the necessity of conforming their own practice to Schenker's theories, they are granting themselves a licence they deny others. " Ibid., 168.

75 « musical intuition guided by long experience »; « the system of underlying knowledge that drives their judgments in individual cases is principled, and that these principles are partly capable of rational articulation »; " as a screen for a set of arbitrary, nonprincipled judgments based more on nepotism than merit. » Ibid., 168. 
La deuxième solution face aux incompatibilités entre théorie et pratique consiste « à imposer une discipline plus stricte en ce qui concerne les procédures et résultats analytiques ${ }^{76}{ }$, solution que Cohn rejette pour les raisons évidentes qu'une telle attitude limite la capacité à produire des analyses intéressantes, identifiée en tant que canon de la richesse par Cohn et Dempster dans la première contribution présentée plus haut.

La troisième solution consiste à « retenir la méthodologie analytique, et la pleine puissance de l'analyse schenkérienne, mais à réévaluer la portée et le statut des visées théoriques ${ }^{77}$. » Cohn opte pour cette solution : il affirme que l'abandon de la prémisse selon laquelle l'Ursatz est la seule source d'unité n'entraîne pas le rejet de Schenker. Il suggère en outre de réorienter le projet schenkérien dans la direction proposée par Nicholas Cook et Christopher Lewis présentée ci-dessus (voir notes 33 et 34), et selon laquelle «la théorie schenkérienne et les théories musicales en général devraient aspirer à la suggestivité des métaphores fortes, plutôt qu'à toutes prétentions ultimes à la vérité ou à la réalité ${ }^{78}$. " Il rappelle en terminant que les schenkériens contemporains n'ont opposé aucune résistance à l'évacuation de la dimension philosophique et épistémologique des méthodes analytiques de Schenker, et que si un tel détachement a été possible, «alors il se pourrait qu'il ne soit pas si préjudiciable de trancher en quelque sorte plus haut dans l'arbre, en isolant [ces mêmes méthodes analytiques] de quelques-uns de leurs principes musicothéoriques ${ }^{79}$. »

La dernière contribution de Cohn, « Schenker's Theory, Schenkerian Theory : Pure Unity or Constructive Conflict ? ${ }^{80}$ est conçue en partie en réponse à la proposition de Nicholas Cook selon laquelle l'analyse schenkérienne traite de «tension, de conflit, d'absence d'unité ». Cohn reconnaît que ce paradigme, qu'il dénomme «Constructive Conflict Paradigm», fait implicitement ou explicitement partie des préoccupations des schenkériens à l'heure actuelle. Toutefois, il met en doute que le concept puisse être attribué à Schenker luimême et démontre son point de vue par une relecture de passages des écrits de ce dernier relatifs à cette question. Il propose plutôt que « la possibilité de l'acceptabilité de conflits dans la théorie schenkérienne résulte du processus

76 « impose stricter discipline on analytic procedures and results. » Ibid., 167.

77 « retain the analytic methodology, and full power of Schenkerian analysis, but reassess the scope and status of the theoretical claims. "Ibid., 169.

78 " Schenkerian theory, and music theories in general, should aim toward the suggestiveness of strong metaphors, rather than any more ultimate claims about truth or reality. " Ibid., 170.

79 « then it might not be so damaging to snip somewhat higher up the tree, by isolating them from some of their music-theoretical tenets. » Ibid., 170.

80 Indiana Theory Review 13, $\mathrm{n}^{\circ} 1$ (1992) : 1-19. 
d'évolution que Rothstein a qualifié d'“américanisation" d'Heinrich Schenker ${ }^{81} »$.

Cohn avance ici que certaines interprétations analytiques reflètent la pénétration dans le discours des schenkériens de thèses que ceux-ci ont fortement critiquées ou, dans le cas de thèses avancées par Eugene Narmour par exemple, rejetées avec véhémence ${ }^{82}$.

Les schenkériens ont été en contact avec les travaux de chercheurs nonschenkériens, tels Meyer, Berry, Narmour, Epstein et Lerdahl et Jackendoff [...] Dans la mesure où [...] [ils] ont exposé leurs positions par rapport à ces travaux, leur accueil n'a pour la plupart pas été favorable ; néanmoins, je propose que ces travaux ont eu un impact sur le développement de la théorie schenkérienne dans les dernières années [...] Il est ironique que les schenkériens aient attaqué non seulement la théorie de Narmour, mais aussi les prémisses métathéoriques que, tel que suggéré, ces derniers partagent avec lui ${ }^{83}$.

Si l'on accepte la thèse développée ici par Cohn, il va sans dire que la pénétration dans la pratique analytique des schenkériens de concepts ouvertement considérés anti-schenkériens complique davantage la lecture de l'appropriation des idées de Schenker. Les trois contributions de Cohn mettent en lumière des aspects importants de ce processus d'appropriation, plus spécifiquement : (1) l'absence de consensus parmi les adhérents à la théorie à l'égard de certains principes fondamentaux; (2) la faille entre la formulation des principes théoriques et la pratique analytique, tant dans les écrits de Schenker que chez les schenkériens contemporains, principalement dans le traitement analytique du motif; (3) les conséquences de l'absence de dialogue au sein de la communauté des chercheurs ; et (4) l'apparition dans la pratique analytique des

81 "the possibility of the acceptability of conflicts in Schenkerian theory results from the evolutionary process that Rothstein has dubbed the " Americanization ”of Heinrich Schenker. ” Ibid., 3-4.

82 Eugene Narmour,Beyond Schenkerism : The Needfor Alternatives in Music Analysis (Chicago : University of Chicago Press, 1977).

83 "Schenkerians have been exposed to the work of non-schenkerians scholars, such as Meyer, Berry, Narmour, Epstein, and Lerdahl \& Jackendoff [...] To the extent that Schenkerians have reacted to this work, their response has, for the most part, not been sympathetic ; nonetheless, I am suggesting that this work has had an impact on the development of Schenkerian theory in recent years [...] The irony is that Schenkerians have attacked not only Narmour's theory, but also those metatheoretical premises which I am suggesting that they come to share with him. » Cohn, «Schenker's Theory, Schenkerian Theory : Pure Unity or Constructive Conflict ? », 13. 
schenkériens contemporains de principes empruntés à une école de pensée dont les prémisses intellectuelles sont notoirement rejetées par ces mêmes praticiens.

\section{Vers une interprétation de l'appropriation des idées de Schenker par les théoriciens-analystes anglo-américains}

La problématique que je désire soumettre maintenant au lecteur a pour objectif de donner un aperçu de la complexité inhérente à l'élaboration d'une interprétation critique du processus d'appropriation à l'étude et, ce faisant, de mettre en évidence la nécessité de poser la question d'une stratégie interprétative adéquate et du recours à des modèles de réflexion utilisés dans les recherches interdisciplinaires.

Avant d'entrer dans le vif du sujet, il est important de préciser que notre propos ne consiste nullement en une évaluation idéologique de la pensée de Schenker, malgré l'urgence de poursuivre le travail entrepris à cet égard par divers chercheurs, mais plutôt en une évaluation des facteurs en jeu dans la construction du discours schenkérien contemporain. Il est notoire que, n'ayant jamais occupé une place centrale dans les débats, les travaux qui explorent les fondements philosophiques et idéologiques de la pensée de Schenker n'ont pas réussi à influencer la pratique des théoriciens-analystes anglo-américains - on pense ici entre autres aux travaux de chercheurs comme Solie, Pastille, Korsyn, Kassler et Deliège ${ }^{84}$. L'absence d'intérêt pour ces contributions témoigne du

84 Ruth Solie, «The Living Work : Organicism and Musical Analysis », 19th-Century Music 4, $\mathrm{n}^{\circ}$ 2 (1980) : 147-56 ; William Pastille, "The Development of the Ursatz in Schenker's Published Work », dans Trends in Schenkerian Research, éd. par Allen Cadwaller (New York : Schirmer, 1990), 71-85; idem, «Heinrich Schenker, Anti-Organicist ", 19th-Century Music 8, $\mathrm{n}^{\circ} 1$ (1984) : 29-36 ; idem, «Ursatz: The Musical Philosophy of Heinrich Schenker » (thèse de doctorat, Cornell University, 1985) ; Kevin Korsyn, «Schenker and Kantian Epistemology », Theoria 3 (1983) : 1-57 ; Jamie Kroy Kassler, « Heinrich Schenker's Epistemology and Philosophy of Music: An Essay on the Relations Between Evolutionary Theory and Music Theory », dans The Wider Domain of Evolutionary Thought, éd. par David Oldroyd et Ian Langham (Dordrecht : R. Reidel Publishing Company, 1983), 221-60 ; Célestin Deliège, Les fondements de la musique tonale (Paris : J. C. Lattès, 1984), 41-49. Certains éléments d'une évaluation idéologique de Schenker ont été mis en évidence dans les contributions commentant la querelle entre Schenker et Schönberg, principalement dans Carl Dahlhaus, « Schoenberg and Schenker », Proceedings of the Royal Musical Association 100 (1973-74) : 209-15 ; Jonathan Dunsby, "Schoenberg and the Writings of Schenker», Journal of the Arnold Schoenberg Institute 2, $\mathrm{n}^{\circ} 1$ (1977) : 26-33 ; et Bryan R. Simms, "New Documents in the SchoenbergSchenker Polemic ", Perspectives of New Music 16, $\mathrm{n}^{\circ} 1$ (1977) : 110-24. Simms conclut son propos (p. 124) en soulignant qu'il est malheureux que nombre des écrits de Schenker aient été traduits et publiés sans ses digressions polémiques, parce que celles-ci « sont essentielles à une juste appréciation de cet esprit créateur complexe » (« because these are central to a balanced understanding of this complex, creative mind »). 
peu d'importance accordée par les praticiens, dans les mots de Deliège, «à l'indispensable discussion critique à laquelle l'œuvre la meilleure doit rester soumise $^{85}$."

\section{Points de convergence et points de divergence}

La présentation des lectures respectives de Nicholas Cook et de Richard Cohn met en évidence des points de convergence de leur interprétation. Tous deux reconnaissent que la fixation des schenkériens sur la démonstration de l'unité, la cohérence des œuvres tonales et, avec elle, la prétention à la rigueur de la vérité scientifique, constituent les principaux obstacles à l'épanouissement de la discipline. Ils proposent tous deux de contrer cette tendance par le recours à l'explication métaphorique. Toutefois, les fondements de leurs argumentations respectives constituent des points de divergence substantiels. Entre autres, Cook affirme qu'une explication possible de l'usage contemporain de la théorie schenkérienne réside dans le fait qu'elle soit comprise comme une théorie de la perception, alors que Cohn l'attribue aux incompatibilités relevées entre la formulation des principes théoriques et la pratique analytique. De plus, quoique Cohn reconnaisse dans certaines contributions récentes l'existence du paradigme «tension, conflit, absence d'unité » proposé par Cook et qu'il désigne «Constructive Conflict», il remet en question l'attribution de ce paradigme à Schenker lui-même, y voyant plutôt une conséquence de l'américanisation de ses idées. Ces quelques points de convergence et de divergence entre les interprétations respectives des deux commentateurs illustrent certes qu'une interprétation de l'appropriation des idées de Schenker se doit d'être entreprise collectivement, mais aussi qu'il peut être extrêmement difficile d'élaborer cette interprétation sans un cadre conceptuel permettant d'intégrer différents points de vue.

Les prises de position de Cook et de Cohn, selon lesquelles l'activité schenkérienne se limite en général à fournir les preuves irréfutables de l'unité de l'œuvre analysée, sont tout à fait justifiées. Cependant, les implications sous-jacentes à la critique de Cook, en ce qui a trait à l'évacuation de la dimension polémique et quasi-philosophique des écrits de Schenker par exemple, ne sont pas pour autant aisément démontrables. En effet, Cook avance que les transformations qu'aura subi le projet initial de Schenker dans le processus d'appropriation ont eu pour conséquence ultime de dénaturer, sinon de falsifier les intentions premières du maître. Que ces transformations soient de l'ordre de la suppression, de l'interprétation, de l'adaptation à des impératifs 
idéologiques au sein de la discipline ou d'un autre ordre, il se trouvera toujours quelqu'un pour arguer que toute entreprise d'appropriation présuppose une médiation et, conséquemment, des transformations, et qu'en fait la théorie de Schenker ne s'en porte que mieux si l'on considère son spectaculaire rayonnement.

À ce point de notre réflexion, $\mathrm{j}$ 'inviterais mon interlocuteur du début à justifier ses résistances face à la perspective de modifications des idées de Schenker pour rendre compte de la spécificité de la musique française en lui posant la question suivante : de quel Schenker s'agit-il donc ? Le Schenker de Der freie Satz, le Schenker censuré de Jonas, le Schenker structuraliste de Forte ou celui des Schachter, Rothgeb, Beach, etc.? Toute lecture critique du discours schenkérien contemporain devrait d'abord et avant tout évaluer si la différence entre les paramètres tels qu'énoncés dans la théorie originale et ceux présentés par les schenkériens des différentes obédiences (orthodoxe, conservateur, néo-schenkérien et postschenkérien) en est une de degré ou de nature.

\section{De la nécessité d'un engagement socio-politique}

Le travail effectué par Cohn, soit la relecture des écrits de Schenker et des principaux adhérents à sa théorie en ce qui concerne la conception schenkérienne du motif, pose des jalons importants dans cette direction et devrait être poursuivi. Il est toutefois crucial, dans le contexte postmoderne de révision en profondeur de l'histoire tant des États-Unis que de l'Europe, d'inscrire le débat dans une perspective socio-politique. Certes, à la suggestion de Cook, la recherche historique sur les sources de la théorie schenkérienne est susceptible de favoriser l'acquisition d'une conscience critique du bagage intellectuel qui leur sont inhérentes. Mais il est impérieux de pousser plus avant la recherche et de faire nôtre le questionnement qui anime les chercheurs en anthropologie sociale et culturelle. C'est seulement de cette façon que nous pourrons éviter de nous enfermer dans une recherche téléologique qui résulterait en une interprétation unique et à l'avance fossilisée du processus d'appropriation des idées de Schenker, centrée principalement sur une comparaison entre la formulation et l'application des éléments constituants de la théorie chez le maître et ses disciples.

Schenker a lui-même présenté sa première contribution substantielle, Harmonielehre, sous le titre de Neue musikalische Theorien und Phantasien, von einem Künstler (Nouvelles théories et fantaisies musicales, par un artiste). Que sont devenues les fantaisies de l'artiste dans les écrits schenkériens contemporains ? Qu'est-il advenu de la spéculation créatrice et du ques- 
tionnement perpétuel, ouvert et ludique, qui caractérisent la liberté avec laquelle Schenker explorait les différents niveaux de structure d'une œuvre, exprimait sa fascination pour telle diminution et suivait diverses pistes d'exploration inscrites dans l'œuvre même ? La virulence de certains de ses propos et le fanatisme du ton n'ont de corollaire que sa passion pour la musique : la passion de celui qui a des convictions personnelles et qui ne sacrifie pas le plaisirde découvrir la pensée des maîtres étudiés à des impératifs idéologiques dictés par un corps institutionnel. Cet état d'esprit représente à mes yeux ce qui devrait constituer l'attitude fondamentale du véritable chercheur. Est-ce le puritanisme anglo-saxon, rigoriste et austère, qui incite les chercheurs à procéder comme si l'accumulation de couches successives de connaissances dites objectives était susceptible de mener, dans quelque mille ans peut-être, à un portrait global et définitif de l'histoire de la tonalité et des œuvres tonales? Espoir absurde s'il en est ! Le discours schenkérien constitue un discours culturel créé, transmis et reçu dans un contexte socio-historique au même titre que tout autre discours culturel. Il faut donc se demander comment l'histoire sociale des États-Unis a contribué à façonner et contribue à perpétuer le discours schenkérien dans sa forme actuelle et, inversement, comment le discours schenkérien contemporain modèle la réalité musicale, universitaire et sociale.

La prise en considération des réalités sociales, psychologiques et institutionnelles qui prévalaient tant lors de l'implantation des idées de Schenker aux États-Unis qu'au cours des différentes phases de leur dissémination soulève plusieurs questions délicates. L'étude de ce contexte signifie en effet celle des positions idéologiques des individus qui l'ont façonné, et ils sont nombreux si l'on considère les 30 années d'efforts déployés, tant à l'origine par les disciples de Schenker émigrés aux États-Unis que par les schenkériens actifs des années 90 .

L' « acquisition », dans les termes de Cook, « d'une conscience critique [...] des structures institutionnelles qui nous incitent en premier lieu à utiliser [les techniques schenkériennes] » (voir note 51), est peut-être l'élément le plus explosif de sa troisième contribution. En effet, une étude critique du processus d'appropriation des idées de Schenker ne peut se faire sans la prise en compte des politiques régissant la vie et le système universitaires américains. Pour en avoir fait partie pendant quelque six ans, $\mathrm{j}$ ' ai pu contaster que le succès des idées de Schenker dans le milieu universitaire repose sur un paradoxe intéressant. D'une part, la théorie de Schenker a réussi à pénétrer la vie universitaire américaine au point d'avoir réduit au silence toute autre approche théorique de la musique tonale. Dans le sillage de cette pénétration est aussi apparue la conviction, pendant au moins une décennie, que cette réalisation était irréversible. 
D'autre part, cette entreprise m'apparaît s'être édifiée à la faveur d'un formidable dialogue de sourds tant entre les membres du groupe qui constitue ses plus farouches adhérents ${ }^{86}$, qu'entre ce même groupe et les critiques, hostiles ou non, aux idées de Schenker qui ont tour à tour proposé modifications, expansions et systématisations ${ }^{87}$. Comme l'a noté Cohn, l'absence d'échanges et de débat public sur la pratique analytique a engendré une situation étonnante dans le contexte d'une discipline qui a tant aspiré à la rigueur scientifique - où les chercheurs « s'accordent une licence qu'ils refusent aux autres » (voir notes 68 et 74), attaquent des prémisses métathéoriques qu'ils en viennent à partager sans toutefois les reconnaître (voir note 83) et, finalement, continuent à éluder toute confrontation, comme le révèle cette observation :

Beach, Rothgeb et Schachter, dans la mesure où ils ont endossé un paradigme de «Constructive Conflict », ou, à tout le moins, reconnu en principe son acceptabilité, n'ont pas indiqué s'ils considèraient ce paradigme comme étant en dernière instance celui mis de l'avant par Schenker ou non. Ce mutisme est particulièrement étonnant si l'on considère que ces chefs de file ont été identifiés pendant plusieurs années à la théorie schenkérienne, non seulement en raison de leur travail analytique, mais aussi de leurs éloges à Schenker, de leurs défenses passionnées de sa théorie lorsqu'attaquée, et, dans certains cas, de leur résistance aux propositions visant à une modification sélective de cette théorie. On s'attendrait à ce que toute distanciation de Schenker concernant un sujet aussi vital que les fondements de sa théorie soit accompagnée de prises de position ouvertes (sinon de justifications scrupuleuses), et provoque un débat salutaire (sinon un vigoureux dissentiment) au sein de la communauté schenkérienne ${ }^{88}$.

86 L'absence de dialogue - sous forme d'analyses comparées d'une même œuvre ou d'examens des séquences décisionnelles menant à un résultat donné dans une analyse - entre Forte et Schachter, entre autres, n'est qu'un exemple parmi tant d'autres.

87 Suite à la parution de Beyond Schenkerism de Eugene Narmour, la stratégie adoptée par les schenkériens consistait en fait à l'ignorer, ou à rejeter, dans les mots de David Beach, tout apport considéré «a thoroughly negative contribution » : "On Analysis, Beethoven, and Extravagance : A Response to Charles Smith ", Music Theory Spectrum 9 (1988) : 174. Voir aussi John Rothgeb, recension de Beyond Schenkerism, Theory and Practice 3, $\mathrm{n}^{\circ} 2$ (1978) : 28-42.

88 « Beach, Rothgeb, and Schachter, to the extent that they have endorsed a Constructive Conflict paradigm, or at least acknowledged its acceptability in principle, have been mute on the question on whether or not they consider this paradigm to be the one ultimately held by Schenker. This muteness is particularly striking in light of the fact that these same leading scholars have been identified with Schenkerian theory for many years, not only in their analytic work, but also in their encomiums to Schenker, their impassioned defenses of his theory under attack, and in some 
Cette absence de dialogue caractérise également la dimension pédagogique de la dissémination des idées de Schenker. Il semble en effet pertinent de poser la question suivante : comment l'intégration des idées de Schenker à l'université, omniprésentes tant au baccalauréat qu'aux études supérieures dans le domaine de la recherche en musique tonale, a-t-elle pu se faire sans le support d'un outil pédagogique puissant, capable de dissiper le silence suspect entourant les difficultés réelles que représente l'analyse des structures intermédiaires d'une œuvre ${ }^{89}$ ?

Que ce soit par honnêteté intellectuelle envers les étudiants ou, plus prosaïquement, pour. consolider, dans les mots de Rothstein, l'entreprise « d'expansion de l'empire schenkérien », l'absence d'un tel outil révèle à quel point les chercheurs évoluent en vase clos. Il est connu que Schenker n'a pas défini rigoureusement certains des termes, des critères et des concepts au cœur de sa théorie; de plus, les procédures qu'il propose ne sont pas explicites. Comment peut-on alors justifier que les schenkériens contemporains n'aient jamais ressenti l'urgence d'un questionnement rigoureux sur les fondements épistémologiques de la théorie schenkérienne depuis les quelque 30 années que dure cette aventure intellectuelle ?Ce questionnement aurait certes pu contribuer à établir le consensus qui fait si cruellement défaut tant chez les chercheurs que chez les étudiants au moment où le prétendu statut d'objectivité de la théorie est sérieusement remis en question.

La règle du non-dit en ce qui a trait aux structures intermédiaires en analyse schenkérienne proclame tout de même, toujours dans les mots de Rothstein, que «backgrounds and middlegrounds are simply not for everybody ${ }^{90}$. Il est beaucoup plus important d'élucider les mécanismes qui ont contribué à circonscrire cet espace intellectuel qu'à déterminer à qui il appartient. Il faut aussi comprendre les mécanismes qui, au sein des structures institutionnelles,

cases their resistance to proposals for selective modification of that theory. One would expect that any distancing from Schenker in as vital a matter as its theoretical underpinnings would be accompanied by open acknowledgements (if not scrupulous justifications), and would provoke healthy debate with (if not vigorous dissent from) other members of the Schenkerian community. " Cohn, « Schenker's Theory, Schenkerian Theory : Pure Unity or Constructive Conflict ? ", 1011.

89 Il serait vain de chercher quelque indication à cet effet dans les deux principales publications à teneur pédagogique en analyse schenkérienne ; voir Allen Forte et Steven E. Gilbert, « Basic Axioms : Structural Levels ; Models of Fundamental Structure », dans Introduction to Schenkerian Analysis (New York : W. W. Norton, 1982), 131-33 ; et David Beach, « Schenker's Theories : A Pedagogical View », dans Aspects of Schenkerian Theory, éd. par David Beach (New Haven : Yale University Press, 1983), 29-32 (section « Advanced Studies in Schenker Analysis »).

90 Rothstein, «The Americanization of Heinrich Schenker », 14. 
orientent les décisions statuant sur la fixité ou la flexibilité du pourtour de cet espace.

La prétention à la rigueur scientifique présuppose à tout le moins la normalisation des procédures explicatives. Qu'enseigne-t-on dans un séminaire en analyse schenkérienne? Un historique de l'évolution de la pensée de Schenker de Harmonielehre à Der freie Satz ou, si l'on tient à former des praticiens, les techniques d'analyse elles-mêmes ? Si l'objectif consiste à former des analystes compétents, une telle absence de consensus sur la transmission des idées de Schenker me semble tout à fait paradoxale.

L'impression dominante est que le schenkérien travaille dans le plus total isolement, animé par la conviction profonde qu'il est sans doute le seul à détenir la « vérité » schenkérienne. Cet isolement serait-il inhérent à la nature même de la discipline de la théorie telle que conçue dans les cercles universitaires américains ? Ceci étant posé, est-ce à dire qu'une théorie de la réception et de l'interprétation des idées de Schenker aux États-Unis serait axée non seulement sur la réception et l'interprétation d'un même phénomène par des agents façonnés par des expériences nationales et culturelles différentes, mais qu'elle se devrait de prendre en compte des agents façonnés par des différences motivées principalement par des intérêts personnels ? Il n'est évidemment pas question de faire appel à cet égard à la psychanalyse pour démêler l'écheveau de l'histoire de l'appropriation et de la réception des idées de Schenker par l'école américaine, ce qui serait ni souhaitable ni, tout compte fait, véritablement fécond.

Toutefois, les intérêts personnels, les préjugés disciplinaires et les enjeux politiques ne peuvent être éludés et leur prise en considération complexifient encore davantage l'exercice d'interprétation. De plus, tel que mentionné plus haut, cette appropriation s'étant effectuée sur une période de plus de 30 ans, ceux qui en étaient à l'origine les principaux agents dans les années 1950-60 sont disparus et ont été remplacés par des nouveaux acteurs et, dès lors, par de nouvelles motivations, et les principaux centres de rayonnement des idées de Schenker se sont eux aussi déplacés et transformés au cours des ans.

Par ailleurs, l'émergence d'une musicologie critique féministe laisse naturellement présager qu'une lecture féministe du phénomène d'appropriation des idées de Schenker - à la fois innovatrice et susceptible de soulever la controverse - verra le jour. Susan McClary a jeté les bases d'une réflexion originale sur les discours théoriques traditionnels en intégrant dans le débat certaines des prémisses caractéristiques de la perspective des musicologues et théoricien(ne)s féministes, plus spécifiquement la question des sexes et de la sexualité. McClary s'intéresse entre autres aux oppositions binaires comme masculin/féminin, actif/passif, fort/faible, normal/anormal, objectif/subjectif, 
que l'on retrouve dans les traités de théorie traditionnelle, plus spécifiquement dans les écrits exposant par exemple les distinctions hiérarchiques entre les thèmes dans la forme sonate, les accords majeur et mineur, les consonances et les dissonances, etc. L'hypothèse selon laquelle l'utilisation d'un langage métaphorique - de métaphores partagées par un groupe social en particulier - contribue à former les catégories métaphysiques qui façonnent la pensée, et, par conséquent, les idéologies sous-jacentes aux discours culturels, invite à une lecture sociologique des écrits de Schenker. Comme le suggèrent les quelques réflexions suivantes tirées de Feminine Endings, McClary propose une interprétation de la pensée schenkérienne encore inexplorée à ce jour. Cette interprétation est susceptible de jeter un tout nouvel éclairage sur l'appropriation des idées de Schenker par les théoriciens-analystes anglo-américains qui ont évacué de son œuvre toute une dimension ou encore l'ont ignorée.

Les sexes et la sexualité n'informent pas que nos théories « abstraites »; la musique elle-même s'appuie souvent fortement sur la simulation métaphorique de l'activité sexuelle pour créer ses effets. Je démontrerai tout au long de ce livre que la tonalité elle-même [...] est le principal moyen musical utilisé durant la période allant de 1600 à 1900 pour éveiller et canaliser le désir [...] Le principal théoricien à reconnaître et à examiner systématiquement cet aspect de la tonalité est Schenker : le but de ses diagrammes quasi mathématiques (en plus de ses tropes à caractère explicitement sexuels) est de relever simultanément les principaux mécanismes en structure profonde par lesquels les compositions tonales éveillent le désir, et les stratégies déployées en surface pour différer la récompense. Au moyen d'un langage théorique et de techniques de notation graphique rigoureux, il relève les mécanismes par lesquels certaines simulations du désir et de la satisfaction sexuels sont constitués au sein du médium musical.

À l'encontre de ses déclarations d'intention mystique, les graphes de Schenker peuvent être interprétés comme étant à même de démontrer en termes pleinement matériels que l'excitation créée dans ces pièces est construite (n'est pas, en d'autres mots, la poursuite du Geist allemand ou de la Volonté de Schopenhauer $)^{91}$.

91 " Not only do gender and sexuality inform our " abstract " theories, but music itself often relies heavily upon the metaphorical simulation of sexual activity for its effects. I will argue throughout this volume that tonality itself $[\ldots]$ is the principal musical means during the period from 1600 to 1900 for arousing and channeling desire [...] The principal theorist to acknowledge and examine this aspect of tonality systematically is Schenker : the purpose of his quasi-mathematical diagrams (in addition to his explicitly sexualized tropes) is to chart simultaneously the principal 
La fréquente utilisation par Schenker de métaphores faisant référence à la sexualité, à la procréation et à la biologie pour présenter certains des concepts de sa théorie, ou encore de substituts analogues pour décrire le fonctionnement de la tonalité, n'a été considérée à ce jour que sous l'angle de l'organicisme. Que les sensations, les affects, les réactions physiques provoquées par la musique soient en effet évacués du discours schenkérien contemporain ne signifie nullement que l'outil analytique ne porte pas dans sa logique interne les ressources qui permettraient d'en rendre compte.

Avant de clore cette section, je poserais volontiers une deuxième question à mon interlocuteur initial : si la théorie de l'universaliste Schenker permet d'analyser toutes les musiques tonales, qu'elles soient de la France, de l'Italie, de la Russie, de la Pologne, etc., alors comment expliquer la quasi totale inexistence de ce répertoire dans les annales de l'activité schenkérienne et dans le canon schenkérien ${ }^{92}$ ? En réponse à cette question, mon interlocuteur pourrait certainement rétorquer que l'exercice n'est pas voué à l'échec parce qu'il n'a pas été tenté. Certes, que certains écrits récents en ethnomusicologie ${ }^{93}$ fassent appel à des principes schenkériens ou quasi schenkériens reflète une

background mechanisms through which tonal compositions arouse desire and the surface strategies that postpone gratification. Through rigorous theoretical language and graphing techniques, he plots out the mechanisms whereby certain simulations of sexual desire and release are constituted within the musical medium. / His mystical statement of intention to the contrary, Schenker's graphs can be read as demonstrating in fully material terms that the excitement achieved in these pieces is constructed (is not, in other words, the tracing of the German Geist or Schopenhauer's Will). » McClary, Feminine Endings, 12-13.

92 Voir David Beach, «A Schenker Bibliography », Journal of Music Theory 13, n 1 (1969) : 2 37, réimprimé dans Readings in Schenker Analysis, éd. par Maury Yeston (New Haven : Yale University Press, 1977) ; idem, "A Schenker Bibliography : 1969-1979 », Journal of Music Theory 23, n० 2 (1979) : 275-86; idem, "The Current State of Schenkerian Research », Acta musicologica 57 (1985) : 275-99; idem, « Schenkerian Theory », Music Theory Spectrum 11, $n^{\circ} 1$ (1989) : 3-14. Voir aussi la bibliographie de Ian Bent et William Drabkin dans Analysis (New York : W. W. Norton, 1987). Pour une liste des œuvres analysées par Schenker, voir Larry Laskowski, Heinrich Schenker : An Annotated Index to His Analyses of Musical Works (New York : Pendragon Press, 1978). On trouvera une bibliographie exhaustive des écrits de et sur Schenker et des écrits d'inspiration schenkérienne jusqu'en 1990 dans Nicolas Meeùs, Heinrich Schenker: une introduction (Liège : Pierre Mardaga, 1993), 91-122. Meeùs offre aussi une évaluation critique très éclairée de certains éléments de la théorie schenkérienne (p. 71-89) de même qu'un glossaire allemand-français et anglais-français (p. 125-26). Meeùs est reponsable de la traduction française de Neue musikalische Theorien und Phantasien III : Der freie Satz (Vienne : Universal Edition, 1935, 1956) sous le titre de L'écriture libre (Liège : Pierre Mardaga, 1993) ; l'ouvrage existe aussi en traduction anglaise dans une édition de Ernst Oster sous le titre de Free Composition (New York : Longman, 1979).

93 Voir Jonathan Stock, «The Application of Schenkerian Analysis to Ethnomusicology : Problems and Possibilities », Music Analysis 12, no 2 (1993) : 215-40 ; et V. Kofi Agawu, "Variation 
volonté d'émancipation de cette approche dogmatique où les excursions à l'extérieur du répertoire restreint que privilégient les praticiens sont à toutes fins utiles inexistantes.

Toutefois, la volonté de mon interlocuteur d'intégrer toutes les musiques tonales au sein du canon schenkérien ne constitue pas en soi une garantie de pertinence. Il importe donc de poser les questions suivantes. Une théorie issue d'une culture musicale donnée garde-t-elle sa pertinence lorsqu'elle est appliquée à des œuvres issues d'une culture musicale différente ? Si tel est le cas, l'analyste qui se penche sur ces œuvres appliquera-t-il les techniques schenkériennes de la même manière qu'il le ferait pour une œuvre du canon schenkérien, en d'autres mots, sans considérations particulières pour l'objet d'étude en question ${ }^{94}$ ? Pour des raisons évidentes d'espace, les problèmes soulevés par ces questions ne peuvent pas être traités ici. Il convient cependant de présenter brièvement quelques éléments d'une réflexion qui est pertinente dans le cadre d'une révision critique du discours schenkérien contemporain.

Prenons l'exemple de la musique française. En réponse à une question sur le concept du développement lors d'un entretien avec Célestin Deliège, Pierre Boulez observait :

On peut trouver une certaine continuité dans la façon dont la tradition allemande a compris la musique, mais il serait très difficile de voir une continuité dans la façon dont les Français l'ont envisagée. En France, si des individus ont compris la musique chacun à leur manière, il n'existe aucune homogénéité de perception, et cela, probablement, parce que les étapes ont été très séparées. $\mathrm{Si}$ on prend les dates, on a Rameau, puis, longtemps après, Berlioz, encore longtemps après, Debussy; vraiment cela ne fait pas une suite. Au contraire, la tradition allemande a vraiment une cohérence continue. Si l'on peut parler d'une continuité dans la tradition française, c'est au XVI ${ }^{\mathrm{e}}$ siècle qu'il faut la trouver. Au XVII siècle, elle s'est pratiquement achevée. Mais au XVII ${ }^{e}$ (disons même entre le $\mathrm{XIII}^{\mathrm{e}}$ et le XVII ${ }^{\mathrm{e}}$ siècle) on trouve une tradition continue de l'écriture ;

Procedures in Northern Ewe Song ", Ethnomusicology 34, nº 2 (1990) :221-43. Les premières excursions à l'extérieur du canon établi par Schenker ont été présentées par Felix Salzer dans Structural Hearing : Tonal Coherence in Music, 2 vol. (New York : Dover Publications, 1962 ; éd. orig., New York: Charles Boni, 1952). Pour un modèle analytique original dérivé de la théorie schenkérienne et traitant d'œuvres hors canon, voir aussi Lori Burns, J. S. Bach's Chorale Harmonizations of Modal Cantus Firmi (thèse de doctorat, Harvard University, 1991), à paraître chez Pendragon Press.

94 Cette problématique a été présentée dans le cadre du colloque « Research Perspectives in Music Scholarship : A Colloquium », à l'Université d'Ottawa, le 26 mars 1994. 
après cela, je ne vois pas du tout, personnellement, de continuité dans la tradition ; à mon sens, je le répète, il est complètement aberrant de parler de tradition française ${ }^{95}$.

La cohérence continue dans la tradition allemande par opposition à l'absence de tradition dans la musique française, dont parle Boulez, ne constitue en rien un point de vue susceptible de provoquer une vive controverse. En effet, le débat autour des conceptions divergentes de la cohérence tonale qui a divisé les écoles musicales d'Occident remonte au XVIII ${ }^{e}$ siècle, soit au moment de la parution de l'œuvre théorique de Jean-Philippe Rameau en Allemagne. Carl Philipp Emanuel Bach a écrit son Versuch über die wahre Art das Clavier zu spielen (Berlin, 1762) ${ }^{96}$ après la publication de tous les ouvrages théoriques de Rameau - en fait, au moment où ceux-ci commençaient à être vraiment pris au sérieux par les théoriciens allemands. En dépit de cette chronologie, il a choisi d'ignorer les données théoriques proposées par Rameau, comme en témoigne ce passage d'une lettre à Kirnberger : « Vous pouvez proclamer haut et fort que mes principes de base et ceux de feu mon père sont contraires à ceux de Rameau ${ }^{97}$. "

Les divergences entre les données proposées par les Bach, père et fils, et par Rameau peuvent être brièvement résumées à ces quelques données fondamentales : Rameau proposait une classification des accords basée sur leur origine verticale alors que la conception allemande reposait sur le comportement de l'accord en relation avec le contexte. En d'autres mots, la conception verticaliste de l'harmonie émane plus directement d'une vision théorique de la grammaire tonale alors que l'école de la basse figurée et du contrepoint d'espèces constituent essentiellement une pratique (par opposition à une théorie) dont les règles ont été codifiées respectivement par Carl Philipp Emanuel Bach et Johann Joseph Fux.

Dans Les fondements de la musique tonale (p. 124), Célestin Deliège observe que les données théoriques proposées à l'époque de Bach et de Rameau "ont engendré deux attitudes à travers l'histoire [...], lesquelles ont eu une profonde incidence sur les pratiques contemporaines et ultérieures et se sont répercutées jusque dans la mentalité des musiciens. » Pour illustrer la nature des divergences profondes qui séparent la tradition austro-allemande et la tradition française, je citerai les propos de Deliège sur le concept de modulation qui sont à mon avis

95 Pierre Boulez, Par volonté et par hasard (Paris : Éditions du Seuil, 1975), 19-20.

96 Trad. et éd. par William J. Mitchell sous le titre de Essay on the True Art of Playing Keyboard Instruments (New York : W. W. Norton, 1949).

97 Cette lettre est citée dans Johann Philipp Kirnberger, Die Kunst des reinen Satzes (Berlin, 1771 [I]; Berlin et Königsberg, 1776-79 [II/1-3]), partie 2, section 3, p. 188. 
plus révélateurs que ne le serait ici l'analyse d'un extrait d'une œuvre de Rameau ou de Berlioz:

Trois noms en France vont suffire à marquer la tonalité : Couperin, dont la conception de la tonalité n'est pas encore absolument ferme et qu'il est préférable de référer au tempérament inégal, Rameau, dont l'idéologie est marquée par la verticalité de l'écriture ; Berlioz, dont la grammaire n'est absolument pas comparable à celle des classiques. Quant à Debussy, il a fait pivoter la musique bien au-delà de la tonalité. Et, s'il se trouve quelque velléitère pour revendiquer César Franck, rappelons-lui la limpide définition de sa technique par Debussy, « une machine à moduler »! Et voilà bien la boutade profonde qui nous mène au cœur du vrai débat. Si Franck est une telle machine, alors, n'en déplaise à mes compatriotes, il est bien le Français d'élection qu'il a choisi d'être. Le concept de modulation, en France, est strictement linéaire, tandis que de Bach à Richard Strauss et Mahler, en Europe centrale, il s'inscrit au même titre que les autres composants de la forme dans une structure hiérarchisée. La pédagogie est révélatrice de cet état d'esprit : on mesure l'écart entre les deux conceptions lorsqu'on lit, par exemple, les données et réalisations des concours d'harmonie du Conservatoire national, publiées avant 1940. C'est en vain que l'on y chercherait l'ombre d'une construction tonale comparable à celle des œuvres de Bach, Haydn, Beethoven, Schubert ou Brahms, pour ne citer que ces noms représentatifs. [...] Aujourd'hui — un peu tard, les besoins ne sont plus les mêmes - on est peut-être en train de dépasser cet état d'esprit, pourtant la conception linéaire de la modulation garde des racines profondes dans les habitudes des enseignants et, par voie de conséquence, des enseignés ${ }^{98}$.

98 Deliège, Les fondements de la musique tonale, 124-25. Cette citation est tirée du chapitre traitant de la modulation où Deliège analyse un extrait du premier mouvement de la Symphonie fantastique, op. 14 (mes. 358-410), analyse fondée sur le modèle schenkérien "amendé ». Paradoxalement, Deliège écrit d'une part : «Berlioz, dont la grammaire n'est absolument pas comparable à celle des classiques [...] » (p. 124); d'autre part, il conclut son analyse en observant: "Sur base d'une telle analyse, se forme une conception de la tonalité, de la modulation et de la cohérence de cet univers, différente, à certains égards, des vues répandues par des traités très populaires, mais plus conforme à la démarche de la tradition des compositeurs classiques d'Europe centrale. » (p. 144). Malgré les laborieuses extensions et modifications des paradigmes schenkériens mises de l'avant par Deliège, son analyse met en évidence une certaine adéquation entre théorie schenkérienne et musique française. Pour une approche analytique de la musique de Berlioz d'inspiration schenkérienne, le lecteur pourra consulter Julian Rushton, The Musical Langage of Berlioz (Cambridge : Cambridge University Press, 1983), 172-74, 202-56. 
La critique de Deliège démontre que la conception française de la modulation est susceptible d'avoir des répercussions profondes sur la définition de la cohérence tonale telle qu'elle se manifeste dans la musique française.

En reconnaissant sa filiation à Brahms, Schönberg situait le développement de sa pensée musicale dans la ligne ininterrompue de l'évolution organique du canon traditionnel cirsconscrit par Bach et Brahms ${ }^{99}$. Ainsi, de Johann Sebastian Bach à Richard Strauss, à Mahler et à Schönberg, les paramètres du discours musical austro-allemand font partie d'une structure hiérarchisée ${ }^{100}$, cette « cohérence continue » dont parle Boulez. On sait que Schenker a élaboré sa théorie principalement à partir d'œuvres de compositeurs allemands et autrichiens, donc à partir d'œuvres qui participent d'une même tradition. Il est aussi notoire qu'il divisait la musique en deux grandes catégories : les chefsd'œuvre, composés par les génies, et la musique des « autres ». La fréquentation de ses écrits nous apprend rapidement que le génie est surtout, sinon exclusivement, d'origine austro-allemande.

De toute évidence, Schenker n'a pas élaboré sa théorie à l'abri dans l'abstrait, soit en dehors des pratiques sociales de son temps et de son propre contexte historique. Là n'est pas la question. Le parti pris nationaliste de Schenker a pour corollaire obligé l'exclusion d'un ensemble d'œuvres tonales de la pratique schenkérienne contemporaine. Lorsque qu'elle est présentée comme la seule approche de la musique tonale faisant autorité, cette pratique distille l'effet pervers d'associer la musique des « autres » à une musique de moindre valeur. Dans une critique des outils musicologiques développés dans le contexte d'un certain canon, Don Michael Randel observait :

Les répertoires pouvant être décrits comme opposant une « résistance à la théorie » viennent facilement à l'esprit. Même le très grand art musical de France et d'Italie, sans parler de l'Angleterre et de l'Espagne, pourrait très bien s'avérer incompatible avec les modèles analytiques développés dans la perspective de démontrer la cohérence des chefs-d'œuvre de certains compositeurs allemands. Cette situation est déplorable seulement si une

99 Arnold Schönberg,Le style et l'idée, traduit de l'anglais par Christiane de Lisle (Paris : Éditions Buchet/Chastel, 1977), 305-43.

100 La démonstration de la continuité dans l'élaboration de la tradition allemande est nulle part plus probante que dans le traité de Schönberg, Fundamentals of Musical Composition (Londres : Faber and Faber, 1970). Schönberg y traite de l'élaboration de cette tradition, comprise comme une totalité, du point de vue du "développement par variation». Quoique plusieurs des exemples musicaux proviennent des sonates pour piano de Beethoven, Schönberg fonde son propos sur des extraits d'œuvres représentant l'ensemble du répertoire austro-allemand. 
telle résistance est utilisée pour laisser croire que cette musique ne mérite pas la plus sérieuse attention que nous pouvons lui accorder en tant que chercheurs $^{101}$.

Ces œuvres tonales qui ne sont pas d'origine austro-allemande font en effet partie du répertoire standard : le répertoire des œuvres préservées et transmises par les institutions dites « de haute culture ». La mise en garde de Randel nous force donc à nous demander, d'une part, comment l'absence d'intérêt pour ces œuvres de la part des praticiens contemporains aura influé sur la construction de l'histoire de la tonalité et sur la perception de ces mêmes œuvres ; et, d'autre part, quelles sont les conséquences de l'évacuation d'une tranche considérable du répertoire tonal sur la discipline - donc, comment s'inscrit l'exclusion d'un ensemble d'œuvres tonales du champ contemporain schenkérien dans le processus d'appropriation des idées de Schenker par les théoriciens-analystes anglo-américains.

Ceci étant posé, s'il y a lieu d'examiner les raisons pour lesquelles les praticiens ne s'aventurent que rarement à l'extérieur du canon établi par Schenker, il est tout aussi important d'examiner les causes profondes de l'absence d'intérêt ou des résistances manifestées par plusieurs chercheurs et théoriciens de langue française, italienne ou espagnole à l'égard du modèle schenkérien, tant dans sa forme originale que dans son interprétation contemporaine. Leur absence quasi totale des débats constitue un élément non négligeable dans l'étude du rayonnement de Schenker aux États-Unis et soulève plusieurs questions qui de toute évidence dépassent le cadre de cet article.

La diversité et la complexité des paramètres en jeu dans la construction du discours schenkérien contemporain démontre à l'évidence que seule une stratégie interprétative puissante pourra rendre compte de la richesse d'un phénomène aussi dense. L'appropriation d'une idée, d'une œuvre d'art, d'une musique ou d'un discours théorique issus d'une culture donnée par une personne, un groupe ou une institution appartenant à une autre culture engendre nécessairement un phénomène de médiation fort complexe. Les sociologues, anthropologues et ethnomusicologues en font leur objet d'étude. Face à la

101 " [It] is quite easy to think of repertoires that could be described as " resistant to theory. "Even the highest art music of France and Italy, to say nothing of England and Spain, might very well prove resistant to analytical methods developed with a view to demonstrating the tonal coherence of the masterpieces of certain German composers. This is unfortunate only if such resistance is translated into the belief that such music does not deserve the most serious attention that we can give it as scholars. » « The Canons in the Musicological Toolbox », dans Disciplining Music, 10-22; 13. 
complexité du phénomène, il semble que seul le recours à des modèles de réflexion développés dans le domaine des sciences sociales et de l'anthropologie culturelle, et repris par les ethnomusicologues, permetttra de formuler une interprétation de la réception et de l'appropriation des idées du théoricien Heinrich Schenker par les théoriciens-analystes anglo-américains.

\section{À l'exemple des ethnomusicologues}

Les recherches en musique populaire ont exposé certaines problématiques qui sont en tous points semblables à celle qui nous intéresse ici. À titre d'exemple, je soumettrai au lecteur quelques-uns des aspects d'une réflexion entreprise par l'ethnomusicologue Jocelyne Guilbault, spécialiste des musiques traditionnelles et populaires des pays créolophones. Cette réflexion vise à démontrer comment l'ethnologue traite l'interprétation des versions contradictoires d'un même phénomène ${ }^{102}$.

Guilbault a étudié la réception et l'appropriation du zouk (genre musical) dans les cinq pays créolophones des Caraïbes (Martinique, Guadeloupe, Dominique, Sainte-Lucie et Haïti) ${ }^{103}$; en d'autres mots, elle s'est penchée sur la réception et l'appropriation d'un même phénomène par des « milieux qui sont façonnés par des expériences sociales, culturelles, politiques et économiques profondément différentes ${ }^{104}$. » Quoique les chercheurs en sciences sociales et en anthropologie aient étudié ces questions avec beaucoup de sérieux et aient développé des stratégies ${ }^{105}$ visant à «donner une voix à des opinions divergentes ${ }^{106}$, ceux-ci n'ont pas considéré l'interprétation de versions

102 Voir Jocelyne Guilbault, Zouk: World Music in the West Indies (Chicago : University of Chicago Press, 1993).

103 Guilbault résume sa stratégie interprétative telle que mise en application dans la deuxième partie de son livre dans « Interpretation out of Contradiction : A World of Music in the West Indies », Canadian University Music Review, n 14 (1994) : 1-17.

104 « in milieus that are shaped by profoundly different social, cultural, political, and economic experiences. " Guilbault, «Interpretation out of Contradiction », 1.

105 Parmi ces stratégies, Guilbault note le mode discursif, le mode dialogique et le texte polyphonique. Elle dirige le lecteur vers quelques-unes des contributions les plus significatives dans le domaine : James Clifford, The Predicament of Culture: Twentieth-Century Ethnography, Literature, and Art (Cambridge : Harvard University Press, 1988) ; James Clifford et George E. Marcus, édit., Writing Culture: The Poetics and Politics of Ethnography (Berkeley: University of California Press, 1986) ; Johannes Fabian,Time and the Other : How Anthropology Makes Its Objects (New York: Columbia University Press, 1983); et George E. Marcus et Michael M. J. Fischer, Anthropology as Cultural Critique : An Experimental Moment in the Human Sciences (Chicago : University of Chicago Press, 1986).

106 « to give a voice to diverging opinions ». Guilbault, « Interpretation out of Contradiction », 2. 
contradictoires d'un même phénomène. Guilbault postule donc sa problématique comme suit: "Comment, au sein de l'écrit ethnologique, les ethnologues peuvent-ils rendre diverses voix manifestes, leur attribuer une présence équivalente à celle de l'auteure alors que, simultanément, celle-ci explicite comment ces voix sont intégrées dans sa propre interprétation ${ }^{107}$ ?»

La stratégie privilégiée par Guilbault consistait à inviter trois spécialistes de son domaine ${ }^{108}$ cumulant trois approches distinctes (pédagogique, académique et quasi-journalistique) à exprimer leur point de vue respectif sur le phénomène du zouk. Le but visé était «d'apprendre de quels points de vue chacun des auteurs considérait le genre [musical] en question et comment chacune de leurs analyses informe les pratiques sociales, culturelles et musicales spécifiques qui constituent le zouk ${ }^{109}$ ». Â la demande de l'ethnologue, le point de vue des spécialistes devait s'inscrire dans un système de référence prédéterminé ${ }^{110}$ et inclure les relations entre le zouk et trois genres musicaux qui ont influé sur son évolution. Guilbault a intégré ces trois contributions à son livre sous la forme de trois chapitres.

L'exercice qui a suivi consistait « à situer les lignes de raisonnement, ou armatures, à la base de chaque contribution et à voir comment celles-ci produisent et organisent les diverses propriétés attribuées au zouk ${ }^{111}$. » L'interprétation de ces trois essais a permis à l'auteur d'identifier l'émergence de points de similarité et surtout d'importants points de divergence sur la manière dont le zouk est perçu ou reçu dans chacune des îles où œuvraient ses collaborateurs.

De ces points de « rencontre », convergents ou divergents, on pouvait éventuellement déduire qu'ils pourraient être considérés comme étant représentatifs de quelques-unes des influences les plus significatives sur

107 « How can various voices be made manifest and given authorial presence at the same time as ethnographers make it clear how these voices are integrated into a personal rendition in ethnographic writing ? "Ibid., 3 .

108 Deux de ces spécialistes, reconnus dans leurs milieux, sont originaires respectivement de la Guadeloupe et de la Dominique, et le troisième est impliqué activement en Haïti depuis plusieurs années.

109 « To learn from which points of view each author addressed the genre in question and how each of their analyses informs the specific social, cultural, and musical practices that constitute zouk. »Ibid., 6.

110 Les paradigmes de ce système de référence sont tirés de Michel Foucault, «Truth and Power », dans Power/Knowledge: Selected Interviews and Other Writings, 1972-1977, by Michel Foucault, éd. par Colin Gordon (New York : Pantheon Books, 1980), 109-33.

111 " To locate the lines of argument, or frameworks, at the foundation of each paper and to see how these produce and organize the various properties attributed to zouk. »Ibid., 8. 
la perception et l'évaluation du zouk dans la région, ou qu'ils pourraient être perçus comme étant représentatifs des éléments focaux dans l'ensemble des relations qui relient le zouk aux trois autres genres ${ }^{112}$.

Guilbault offre en appendice de son article une liste des éléments focaux qui ont « orienté la description des caractéristiques du zouk dans chacun des trois essais. » Cet appendice, à la fois extrêmement dense et clairement structuré, " définit les divers types de liens (linguistique, politique, économique, historique, musical, racial, géographique, contextuel) qui caractérisent ce champ musical circonscrit historiquement et socialement ${ }^{113}$.»

Ces démonstrations et conclusions sont particulièrement intéressantes pour quiconque s'intéresse à l'interprétation de l'appropriation des idées de Schenker par les théoriciens-analystes anglo-américains. En effet, quoique cette appropriation implique un groupe d'individus aux caractéristiques apparemment semblables - jusqu'à ce que les représentants britanniques se joignent au groupe de chercheurs en analyse schenkérienne, les membres de ce groupe étaient tous des résidents des États-Unis, universitaires et soumis à des déterminismes institutionnels relativement identiques sur une période de temps donnée - , les multiples éléments en jeu tissent une trame extrêmement dense, comme les observations ci-dessus tentent de le démontrer.

Une lecture éclairée de ce phénomène complexe doit faire appel aux développements théoriques mis de l'avant par les chercheurs de diverses disciplines, y compris l'ethnomusicologie. Si le lecteur veut bien substituer la théorie schenkérienne au zouk de Guilbault et, de même, remplacer les trois genres musicaux qui ont influé sur l'évolution du zouk par trois interprétations contemporaines qui ont contribué à modifier la pensée de Schenker, il sera à même d'apprécier la validité et la pertinence du recours à l'interdisciplinarité pour développer une stratégie permettant de rendre compte de l'appropriation des idées de Schenker par les théoriciens-analystes anglo-américains. Et, cela va sans dire, il pourra goûter l'ironie inhérente à l'entreprise d'une lecture ethnologique dont l'objet d'étude est un discours qui est encore un discours

112 «One possible conclusion that could be deduced from these "meeting" points, whether converging or diverging, was that they could be considered representative of some of the most significant influences on the perception and evaluation of zouk in the region or seen as representative of the focal elements in the ensemble of relations that link zouk with the three other genres. »Ibid., 10.

113 «that oriented the description of zouk's characteristics in the three essays »; " defines the diverse kinds of links (linguistic, political, economic, historical, musical, racial, geographic, contextual) that characterize this historically and socially located musical field. » Ibid., 10. L'appendice en question se trouve à la page 16 . 
dominant et qui, par surcroît, est issu d'une culture impérialiste, elle aussi dominante.

\section{Résumé}

Cette étude présente en premier lieu certains des éléments qui caractérisent les nouveaux espaces de réflexion en musicologie et en théorie de la musique. Puis, après avoir situé l'évolution récente de la théorie schenkérienne dans le panorama intellectuel, l'auteure expose une problématique qui vise à mettre en évidence la nécessité d'une interprétation éclairée de la réception et de l'appropriation des idées du théoricien autrichien Heinrich Schenker par les théoriciens-analystes anglo-américains. L'auteure suggère que la diversité et la complexité des paramètres en jeu dans la construction du discours schenkérien contemporain démontrent à l'évidence que seule une stratégie interprétative puissante pourra rendre compte de la richesse d'un phénomène aussi dense. Elle conclut en proposant que, face à la complexité de ce phénomène, il semble que seul le recours à des modèles de réflexion développés dans le domaine des sciences sociales et de l'anthropologie culturelle, et repris par les ethnomusicologues, permetttra de formuler une révision critique du discours schenkérien. 